\title{
Orlicz spaces of finitely additive set functions*
}

\section{by}

\section{J. J. UHL, Jr. (Pittsburgh, Penn.)}

A serious study of finitely additive set functions appears to have started during the last part of the last century with, for instance, Jordan content. Though the Borel-Lebesgue theory of countably additive set functions has eclipsed the earlier work mostly through the first quarter of this century, the representation theory of linear functionals on spaces of bounded functions revived interest in finitely additive set functions in the early thirties, and the abstract integration relative. to finitely additive measures developed extensively in the next decade. More important is the realization that finitely additive set functions provide considerable flexibility in many applications.

In probability theory, Dubins and Savage [8] have noted that countable additivity is a restrictive hypothesis and have dropped it. Even in the study of classical function spaces such as $L^{p}$ Leader [13] finds it "natural to consider" the " $L^{p}$-spaces of finitely additive set functions" called the $\nabla^{p}$-spaces. Motivated by these considerations, we shall consider spaces of set functions which are more general that the $\nabla^{p}$-spaces but which also are endowed with an interesting structure.

Section $I$ is devoted to defining Orlicz spaces $\left(V^{\Phi}\right)$ of finitely additive set functions having their values in a Banach space. In section II, it is shown that if $L^{\Phi}$ is the corresponding Orlicz space of point functions, there exists an isometric injection of $L^{\Phi}$ into $V^{\Phi}$. The $V^{\Phi}$-spaces generalize Leader's $V^{p}$-spaces of real finitely additive functions in much the same way the Orlicz spaces $L^{\phi}$ generalize the Lebesgue spaces $L^{p}$. At the root of Leader's work is the Radon-Nikodym-Bochner theorem ([9], IV. 9. 14) which is available only in the scalar case. An extension of this theorem for vector-valued set functions is proved and then applied in sections III and IV analyzing the structure of $\nabla^{\Phi}$-spaces. In section $V$ a representation

* This paper contains some of the main results of the author's doctoral dissertation written at Carnegie Institute of Technology under the guidance of Professor M. M. Rao to whom the author expresses his sincerest thanks.

This research was supported in part by NSF Grant 55183 and Army Grant 50029. 
of bounded linear operators on certain $V^{\infty}$-spaces to an arbitrary Banach space is obtained and the space of such operators is characterized. This result is specialized to $L^{\Phi}$ in representing all bounded linear operators of $L^{\Phi}$ into any Banach space whenever $\Phi$ obeys a growth condition. Some of the results contained in this paper are announced in [21] without proof.

\section{The $V^{\Phi}(X)$ - spaces}

The basis for this section is finitely additive oxtended real-valued non-negative set function $\mu$ defined on a field $\Sigma$ of subsets of a point set $\Omega . \Sigma_{0} \subset \Sigma$ is the ring of sets of finite $\mu$-measure. Various Banach spaces of finitely additive set functions having their values in a real or complex Banach (or $B$-) space $\mathscr{X}$ will be defined and investigated.

Let $\varphi$ be a left continuous non-decreasing real-valued function which does not vanish identically and satisfies $p(0)=0$. Let $\psi$ denote the left continuous inverse of $\varphi$ defined by the following convention: If $\phi$ is discontinuous at $a$, then $\psi(v)=a$ for $\varphi(a-)<v \leqslant \varphi(a+)$, and if $\varphi(u)=0$ for $a<u \leqslant b$ but $\varphi(u)<c$ for $u<a$, then $\varphi(c)=a$. If $\lim \varphi(u)=l$ is finite, then $\psi(v)=\infty$ for $v>l$. By this convention, $\psi(0)=0$ and $\psi$ is well-defined on the positive line. $p$ and $\psi$ are "generalized inverses" to each other and are mutually inverse to each other whenever both are strictly increasing and continuous.

Definition 1 . If the non-decreasing functions $\phi$ and $\psi$ are mutually inverse, and satisfy the above conditions, then the functions $\Phi$ and $\Psi$ defined on the line by the Lebesgue integrals

$$
\Phi(u)=\int_{0}^{|u|} \varphi(t) d t, \quad \Psi(v)=\int_{0}^{|v|} \psi(t) d t
$$

are called complementary Young's functions. A Young's function $\Phi$ obeys the $\Delta_{2}$-condition if there exists $K<\infty$ such that $\Phi(2 x) \leqslant K \Phi(x)$ for all $x$.

It follows that $\Phi$ and $\Psi$ are convex, increasing, and are continuous except for at most one point, after which the function must be identically $\infty$. These facts and the proof of the following proposition are well-known ([24], p. 77).

Proposition 2 (Young's inequality). If $\Phi$ and $\Psi$ are complementary Young's functions, then

$$
|x y| \leqslant \Phi(x)+\Psi(y)
$$

for all real numbers $x$ and $y$. Equality holds if and only if one of the relations $y=\varphi(x)$ or $x=\psi(y)$ is satisfied.
By convention, throughout this paper, $\Phi$ and $\Psi$ will denote (nontrivial) Young's functions.

Definition 3 . If $E \in \Sigma$, a partition $\pi$ of $E$ is any finite disjoint eollection $\left\{E_{n}\right\} \subset \Sigma_{0}$ satisfying $\bigcup_{n} E_{n} \subset E$. The partitions of a set $E$ are partially ordered by defining $\pi_{1} \leqslant \pi_{2}$ whenever each element of $\pi_{1}$ is a union of elements of $\pi_{2}$. In this case $\pi_{2}$ is said to be a refinement of $\pi_{1}$

It may be noted that $\pi=\left\{E_{n}\right\}$ may be a partition of $\Omega$, as defined above, without satisfying the relation $\Omega=\bigcup_{n} E_{n}$. This latter condition can hold only if $\mu(\Omega)$ is finite.

Definition 4. Let $\Phi$ be a Young's function, $\mathscr{X}$ a $B$-space, and $F$ a finitely additive $\mathscr{X}$-valued set function defined on and vanishing on $\mu$-null sets. If $E \in \Sigma, I_{\Phi}(F, E)$ is defined by

$$
I_{\Phi}(F, E)=\sup _{\pi} \sum_{\pi} \Phi\left(\frac{\left\|F\left(E_{n}\right)\right\|}{\mu\left(E_{n}\right)}\right) \mu\left(E_{n}\right)
$$

where the supremum is taken over all partitions $\pi=\left\{E_{n}\right\}$ of $E$. (Here the convention $0 / 0=0$ is observed and will be used throughout.)

For simplicity of notation, the function $I_{\sigma}(\cdot)$ will be applied to either vector-valued set functions; the meaning will be clear from the context. Also $I_{\Phi}(F, \Omega)$ will be written as $I_{\Phi}(F)$. It is clear that $I_{\Phi}(F, E)$ is unambiguously defined as a finite number or as $+\infty$. If $I_{\Phi}(F)$ is finite, $F$ is said to be of $\Phi$-bounded variation. When $\Phi(x)=x^{p}, p \geqslant 1$, the notion of $\Phi$-bounded variation reduces to the well-known notion of $p$-bounded variation. This and the $\Phi$-bounded variation are sometimes referred to as the "Hellinger and the generalized Hellinger" integrals of $F$ when $F$ and $\mu$ are real-valued and countably additive [15].

LEMma 5. For each $F$ such that $I_{\Phi}\left(F^{\prime}\right)$ exists,

$$
\sum_{\pi} \Phi\left(\frac{\left\|F\left(E_{n}\right)\right\|}{\mu\left(E_{n}\right)}\right) \mu\left(E_{n}\right)
$$

is a non-decreasing function of the partitions $\pi$ of $\Omega$. Consequently

$$
I_{\Phi}(F, E)=\lim _{\pi} \sum_{\pi} \Phi\left(\frac{\left\|F\left(E_{n}\right)\right\|}{\mu}\right) \mu\left(E_{n}\right)
$$

where the limit is taken in the Moore-Smith sense [10] through all partitions of $E \in \Sigma$.

Proof. It is sufficient to show that

$$
\Phi\left(\frac{\|F(A \cup B)\|}{\mu(A \cup B)}\right) \mu(A \cup B) \leqslant \Phi\left(\frac{\|F(A)\|}{\mu(A)}\right) \mu(A)+\Phi\left(\frac{\|F(B)\|}{\mu(B)}\right) \mu(B)
$$


whenever $A, B \in \Sigma_{0}, A \cap B=\emptyset$. If either $\mu(A)$ or $\mu(B)=0$, then the inequality is true and trivial. So, suppose $\mu(A), \mu(B)>0$. The monotonicity and convexity of $\Phi$ imply

$$
\begin{aligned}
\Phi\left(\frac{\|F(A \cup B)\|}{\mu(A \cup B)}\right) & \leqslant \Phi\left(\frac{\left\|F^{\prime}(A)\right\|+\left\|F^{\prime}(B)\right\|}{\mu(A)+\mu(B)}\right) \\
& \leqslant \frac{\mu(A)}{\mu(A)+\mu(B)} \Phi\left(\frac{\|F(A)\|}{\mu(A)}\right)+\frac{\mu(B)}{\mu(A)+\mu(B)} \Phi\left(\frac{\left\|F^{\prime}(B)\right\|}{\mu(B)}\right) .
\end{aligned}
$$

Hence,

$\Phi\left(\frac{\left\|F^{\prime}(A \cup B)\right\|}{\mu(A \cup B)}\right) \mu(A \cup B) \leqslant \Phi\left(\frac{\|F(A)\|}{\mu(A)}\right) \mu(A)+\Phi\left(\frac{\left\|F^{\prime}(B)\right\|}{\mu(B)}\right) \mu(B)$, q.e.d.

LEMra 6. $I_{\Phi}(F, \cdot)$ is a finitely additive set function on $\Sigma$ and vanishes on $\mu$-null sets.

Proof. The second statement is evident only the first needs a proof. Let $S_{1}, S_{2} \in \Sigma, S_{1} \cap S_{2}=\varnothing$. If $\pi_{1}$ and $\pi_{2}$ are arbitrary partitions of $S_{1}$ and $S_{2}$ respectively, then $\pi_{1} \cup \pi_{2}$ is a partition of $S_{1} \cup S_{2}$. From the definition of $I_{\Phi}(F, \cdot)$,

$$
\begin{aligned}
I_{\Phi}\left(F, S_{1} \cup S_{2}\right) & \geqslant \sum_{\pi_{1} \cup \pi_{2}} \Phi\left(\frac{\left\|F\left(\mathbb{E}_{n}\right)\right\|}{\mu\left(\mathbb{E}_{n}\right)}\right) \mu\left(\mathbb{E}_{n}\right) \\
& =\sum_{\pi_{1}} \Phi\left(\frac{\left\|F\left(E_{n}\right)\right\|}{\mu\left(E_{n}\right)}\right) \mu\left(E_{n}\right)+\sum_{\pi_{2}} \Phi\left(\frac{\left\|F\left(\mathbb{E}_{n}\right)\right\|}{\mu\left(\mathbb{E}_{n}\right)}\right) \mu\left(\mathbb{E}_{n}\right) .
\end{aligned}
$$

Taking suprema on both sides of this inequality over all partitions $\pi_{1}$ of $S_{1}$ and $\pi_{2}$ of $S_{2}$, we obtain

$$
I_{\Phi}\left(F, S_{1} \cup S_{2}\right) \geqslant I_{\Phi}\left(F, S_{1}\right)+I_{\Phi}\left(F, S_{2}\right) .
$$

To prove the reverse inequality, let $\pi=\left\{E_{n}\right\}$ be an arbitrary partition of $S_{1} \cup S_{2}$. Then $\pi_{1}=\left\{E_{n} \cap S_{1}\right\}$ and $\pi_{2}=\left\{E_{n} \cap S_{2}\right\}$ aro partitions of $S_{1}$ and $S_{2}$ respectively, and $\pi_{1} \cup \pi_{2}$ is a refinement of $\pi$. According to lemma 5 , and the definition of $I_{\Phi}(F, \cdot)$,

$$
\begin{aligned}
& \qquad \sum_{\pi} \Phi\left(\frac{\left\|F\left(E_{n}\right)\right\|}{\mu\left(\mathbb{E}_{n}\right)}\right) \mu\left(\mathbb{E}_{n}\right) \\
& \leqslant \sum_{\pi_{1}} \Phi\left(\frac{\left\|F\left(E_{n} \cap S_{1}\right)\right\|}{\mu\left(E_{n} \cap S_{1}\right)}\right) \mu\left(E_{n} \cap S_{1}\right)+\sum_{\pi_{2}} \Phi\left(\frac{\left\|F\left(E_{n} \cap S_{2}\right)\right\|}{\mu\left(\mathbb{E}_{n} \cap S_{2}\right)}\right) \mu\left(\mathbb{E}_{n} \cap S_{2}\right) \\
& \leqslant I_{\Phi}\left(F, S_{1}\right)+I_{\Phi}\left(F, S_{2}\right) . \\
& \quad \text { Consequently } \\
& \quad I_{\Phi}\left(F, S_{1} \cup S_{2}\right)=I_{\Phi}\left(F, S_{1}\right)+I_{\Phi}\left(F, S_{2}\right), \quad \text { q.e.d. }
\end{aligned}
$$

The next theorem shows that $I_{\Phi}(\cdot)$ is lower semi-continuous.

THEOREM 7. Let $\left\{G_{\tau}, \tau \epsilon T\right\}$ be a net of set functions on $\Sigma_{0}$ such that $I_{\Phi}(G)$ is defined for each $\tau \in T$ and $\lim G_{\tau}(E)=G(E)$ in the weak topology of $\mathscr{X}$ for each $E \in \Sigma_{0}$. Then $I_{\Phi}(G, E)$ is defined and

for each $E \in \Sigma$.

$$
I_{\Phi}(G, E) \leqslant \liminf _{\tau} I_{\Phi}\left(G_{\tau}, E\right)
$$

Proof. Without loss of generality, it may be assumed that $E=\Omega$. If is clear that the function $G$ defined above on $\Sigma_{0}$ vanishes on $\mu$-null sets. It is also immediate that $G$ is finitely additive on $\Sigma_{0}$, so that $I_{\Phi}(G)$ is well defined.

Now, whether $\Phi$ is continuous or discontinuous (i.e. $\Phi$ jumps to $+\infty$ at some point),

$$
\Phi\left(\frac{\|G(E)\|}{\mu(E)}\right) \leqslant \liminf _{\tau} \Phi\left(\frac{\left\|G_{\tau}(E)\right\|}{\mu(E)}\right)
$$

for each $E \epsilon \Sigma_{0}$ since $\|G(E)\| \leqslant \liminf \left\|G_{\tau}(E)\right\|$ ([9], II. 3.27). Therefore, if $\pi_{0}=\left\{E_{n}\right\}$ is any partition of $\Omega$, the following chain of inequalities holds :

$$
\begin{aligned}
\sum_{\pi_{0}} \Phi\left(\frac{\left\|G\left(E_{n}\right)\right\|}{\mu\left(E_{n}\right)}\right) \mu\left(E_{n}\right) & \leqslant \sum_{\pi_{0}} \liminf _{\tau} \Phi\left(\frac{\left\|G_{\tau}\left(E_{n}\right)\right\|}{\mu\left(E_{n}\right)}\right) \mu\left(E_{n}\right) \\
& \leqslant \underset{\tau}{\liminf } \sum_{\pi_{0}} \Phi\left(\frac{\left\|G_{\tau}\left(E_{n}\right)\right\|}{\mu\left(E_{n}\right)}\right) \mu\left(E_{n}\right) \\
& \leqslant \underset{\tau}{\liminf I_{\Phi}\left(G_{\tau}\right), \quad \text { q.e.d. }}
\end{aligned}
$$

In integration theory, much of the structure of spaces of integrable functions is based on the behavior of simple functions, that is, functions which assume only finitely many values. Our work, too, depends to a certain extent on the behavior of indefinite integrals of simple functions.

Definition 8. If $\pi=\left\{E_{n}\right\}$ is a partition of $\Omega$, a set function of the form $\sum_{\pi} a_{n} \mu \cdot E_{n}, a_{n} \in \mathscr{X}$, is called a step function. Here $\mu \cdot E_{n}$ is the set function $\mu\left(E_{n} \cap \cdot\right)$. Of special interest are step functions of the form

$$
\sum_{n} \frac{F\left(E_{n}\right)}{\mu\left(E_{n}\right)} \mu \cdot E_{n}
$$

where $F$ is finitely additive on $\Sigma_{0}$ and $\pi$ is a partition of $\Omega$. Leader [13] has termed such a function the projection of $F$ on $\pi$. The projection of $F$ on $\pi$ will be denoted by $F_{\pi}$. 
THEOREM 9. If $I_{\Phi}(F)$ is defined and $\pi=\left\{E_{n}\right\}$ is any partition of $\Omega$ then

$$
I_{\Phi}\left(F_{\pi}\right)=\sum_{\pi} \Phi\left(\frac{\left\|F^{\prime}\left(E_{n}\right)\right\|}{\mu\left(E_{n}\right)}\right) \mu\left(E_{n}\right) .
$$

Consequently, $I_{\Phi}\left(F_{n}\right) \leqslant I_{\Phi}(F)$, and $I_{\Phi}(F, E)=\lim I_{\phi}\left(F_{\pi}^{\prime}, E\right)$.

Proof. A brief computation shows that if $\pi_{0}=\left\{W_{n}\right\}$ is any partition and $\pi=\left\{S_{m}\right\} \geqslant \pi_{0}$, then

$$
\sum_{\pi} \Phi\left(\frac{\left\|F_{\pi}\left(S_{m}\right)\right\|}{\mu\left(S_{m}\right)}\right) \mu\left(S_{m}\right)=\sum_{\pi_{0}} \Phi\left(\frac{\left\|F\left(W_{n}\right)\right\|}{\mu\left(H_{n}\right)}\right) \mu\left(H_{n}\right)
$$

The remaining assertions of the theorem follow from lemma 5, a.e.d. The following definitions and theorems are devoted to the introduction of a class of Banach spaces of $\mathscr{X}$-valued set functions. When $\mu$ is countably additive and has the finite subset property, Rao [18, 19] has shown that some of these spaces are equivalent to conjugate spaces of certain Orlicz spaces.

Definition 10. $A^{\Phi}(\Omega, \Sigma, \mu, \mathscr{X})\left(=A^{\Phi}(\mathscr{X})\right)$ consists of all finitely additive $\mathscr{X}$-valued set functions $F$ on $\Sigma_{0}$ satisfying

(i) In vanishes on $\mu$-null sets;

(ii) $I_{\Phi}(F / K) \leqslant 1$ for some $K>0$.

Using the convexity of $\Phi$, one ean easily show that whenever $I_{\Phi}\left(I^{\prime}\right)$ $\leqslant \infty, F \in A^{\Phi}(\mathscr{X})$. By $\overparen{A^{\Phi}(\mathscr{X})}$ is meant $\left\{F: I_{\Phi}\left(F^{H}\right)<\infty\right\}$. If $\Phi$ obeys the $\Delta_{2}$-condition, then $A^{\Phi}(\mathscr{X})=\overparen{A^{\Phi}(\mathscr{X})}$. To see this, choose $p$ such that $I_{\Phi}\left(F / p_{0}\right) \leqslant 1$ and a positive integer $n$ such that $2^{n} \mid p \geqslant 1$. The monotonicity of $\Phi$ and the $\Delta_{2}$-condition yield

$$
I_{\Phi}\left(F^{\prime}\right) \leqslant I_{\Phi}\left(\frac{2^{n}}{p} F\right) \leqslant K^{n} I_{\Phi}\left(\frac{F}{p}\right) \leqslant K^{n}<\infty .
$$

In the next theorem, a norm on $A^{\Phi}(\mathscr{X})$ is introduced relative to which $A^{\Phi}(\mathscr{X})$ becomes a Banach space.

THeorem 11. The functional $N_{\phi}\left(H^{\prime}\right)=\inf \left\{k>0: I_{\infty}\left(F^{\prime} / K\right) \leqslant 1\right\}$ on $A^{\Phi}(\mathscr{X})$ is a norm under which $A^{\Phi}(\mathscr{X})$ is a Banach space.

Proof. (i) $N_{\phi}\left(F^{\prime}\right)=0$ if and only if $H^{\prime}(E)=0$ for all $E \in \Sigma_{0}$, then $I_{\Phi}(F / k)=0$ for all $k>0$. Hence $N_{\phi}\left(F^{\prime}\right)=0$. Oonversely suppose there exists $F^{\prime} \in A^{\Phi}(\mathscr{X})$ with $N_{\Phi}\left(F^{\prime}\right)=0$ and such that $F^{\prime}(E) \neq 0$ for some $\mathscr{E} \in \Sigma_{0}$. Form the (trivial) partition $\pi=\{T\}$. Since $N_{\phi}\left(F^{\prime}\right)=0, I_{\Phi}\left(T^{\prime} / l\right) \leqslant 1$ for all $k>0$. From lemma 5 , it follows that $\Phi\left(\left\|W^{\prime}(E)\right\| / k \mu(E)\right) \mu(E)<1$ for all $k>0$. On the other hand, because $\lim \Phi(x)=+\infty$, $\lim _{k \rightarrow 0} \Phi\left(\left\|H^{\prime}(E)\right\| / k \mu(E)\right) \mu(E)=\infty$, a contradiction. (ii) $N_{\Phi}\left(a F^{\prime}\right)=|a| N_{\Phi}(F)$ for all scalars $a$ and all $F$ in $A^{\Phi}(\mathscr{X})$. The equality holds trivially if $a=0$. When $a \neq 0$,

$$
\begin{aligned}
N_{\Phi}\left(a F^{\prime}\right) & =\inf \left\{k>0: I_{\Phi}\left(\frac{a F}{k}\right) \leqslant 1\right\} \\
& =\inf \left\{k>0: I_{\Phi}\left(F / \frac{k}{|a|}\right) \leqslant 1\right\} \\
& =\inf \left\{|a| t>0: I_{\Phi}\left(\frac{F}{t}\right) \leqslant 1\right\} \\
& =|a| N_{\Phi}(F) .
\end{aligned}
$$

(iii) $N_{\Phi}\left(F_{1}+F_{2}\right) \leqslant N_{\Phi}\left(F_{1}\right)+N_{\Phi}\left(F_{2}\right)$ for all $F_{1}, F_{2} \in A^{\Phi}(\mathscr{X})$. From the convexity and monotonicity of $\Phi$, it follows that, provided neither $F_{1}$ nor $F_{2}=0$

$$
\begin{aligned}
& I_{\Phi}\left(\frac{F_{1}+F_{2}}{N_{\Phi}\left(F_{1}\right)+N_{\Phi}\left(F_{2}\right)}\right) \\
& \quad \leqslant \frac{N_{\Phi}\left(F_{1}\right)}{N_{\Phi}\left(F_{1}\right)+N_{\Phi}\left(F_{2}\right)} I_{\Phi}\left(\frac{F_{1}}{N_{\Phi}\left(F_{1}\right)}\right)+\frac{N_{\Phi}\left(F_{2}\right)}{N_{\Phi}\left(F_{1}\right)+N_{\Phi}\left(F_{2}\right)} I_{\Phi} \frac{F_{2}}{N_{\Phi}\left(F_{2}\right)} \\
& \quad \leqslant \frac{N_{\Phi}\left(F_{1}\right)+N_{\Phi}\left(F_{2}\right)}{N_{\Phi}\left(F_{1}\right)+N_{\Phi}\left(F_{2}\right)}=1 .
\end{aligned}
$$

Hence $N_{\Phi}\left(F_{1}+F_{2}\right) \leqslant N_{\Phi}\left(F_{1}\right)+N_{\Phi}\left(F_{2}\right)$. If either $F_{1}=0$ or $F_{2}=0$, the inequality is true and trivial.

(iv) $A^{\Phi}(\mathscr{X})$ is complete under $N_{\Phi}(\cdot)$. Suppose $\left\{F_{n}\right\} \subset A^{\Phi}(\mathscr{X})$ is a Cauchy sequence. The definition of $N_{\Phi}(\cdot)$ establishes the existence of a double sequence $\left\{N_{n, m}\right\}$ of positive members such that

$$
\lim _{n, m} N_{n, m}=+\infty \quad \text { and } \quad I_{\Phi}\left(N_{n, m}\left(\boldsymbol{F}_{n}-F_{m}\right)\right) \leqslant 1
$$

for all positive integers $m$ and $n$. First it will be shown that $\lim \boldsymbol{F}_{n}(E)$ exists in the strong topology of $\mathscr{X}$ for each $E \epsilon \Sigma_{0}$. If $\mu(E)=0$, then $F_{n}(E)$ $=0$ for all $n$. If $\mu(E)>0$, the definition of $I_{\Phi}(\cdot)$ implies

$$
\Phi\left(\frac{N_{n, m}\left\|F_{n}(E)-F_{m}(E)\right\|}{\mu(E)}\right) \mu(E) \leqslant I_{\Phi}\left(N_{n, m}\left(F_{n}-F_{m}\right)\right) \leqslant 1
$$

for all $m$ and $n$. Since $\lim N_{n, m}=\infty$ and $\lim \Phi(x)=\infty$, one has $\lim _{n, m}\left\|F_{n}(E)-F_{m}(E)\right\|=0$. Since $\mathscr{X}$ is a Banach space, the set function $F$ defined for each $E$ in $\Sigma_{0}$ by

$$
F(E)=\lim _{n} F_{n}(E)
$$

is well-defined and vanishes on $\mu$-null sets. It is evident that $F$ is additive. 
Next it will be shown that $F_{\in} \in A^{\Phi}(\mathscr{X})$ and $\lim _{n} N_{\Phi}\left(F_{n}-F^{\prime}\right)=0$. The fact that $\lim N_{\Phi}\left(F_{n}-F_{m}\right)=0$ and the triangle ineguality imply $\varrho=\lim _{n} N_{\Phi}\left(F_{n}\right)$ exists. If $\varrho=0$, then $F=0$ and $\lim _{n} N_{\Phi}\left(F_{n}-F^{\prime}\right)=0$, and the proof is finished. If $\varrho \neq 0$, it may be assumed that $N_{\Phi}\left(F_{n}^{\prime}\right) \neq 0$ for all $n$. Then

$$
\lim _{n} \frac{F_{n}\left(W^{\prime}\right)}{N_{\Phi}\left(F_{n}\right)}=\frac{H^{\prime}(l i)}{\varrho}
$$

strongly in $\mathscr{X}$ for each $\mathbb{E} \epsilon \Sigma_{0}$. The lower semi-continuity of $I_{\Phi}$ (Lemma 7) guarantees

$$
I_{\Phi}\left(\frac{F^{\prime}}{\varrho}\right) \leqslant \liminf _{n} I_{\Phi}\left(\frac{H_{n}^{\prime}}{N_{\Phi}\left(F_{n}\right)}\right) \leqslant 1
$$

Hence $F^{\prime} \in A^{\Phi}(\mathscr{X})$. Now let $K>0$ be arbitrary and $P_{K}$ bo chosen so that $N_{n, m} \geqslant K$ and for $n, m \geqslant P_{K}$. For each $n$ and $m$ the monotonicity of $\Phi$ yields $I_{\Phi}\left(K\left(F_{n}-F_{m}\right)\right) \leqslant I_{\Phi}\left(N_{n, m}\left(F_{n}^{\prime}-F_{m}\right)\right) \leqslant 1$. Another application of the lower semi-continuity (theorem 7) of $I_{\infty}(\cdot)$ and the definition of $F$ yield

$$
I_{\Phi}\left(K\left(H_{m}-F^{\prime}\right)\right) \leqslant \liminf _{\Phi}\left(K\left(F_{m}-F_{n}\right)\right) \leqslant 1 \quad \text { for } \quad m \geqslant P_{K \Sigma} .
$$

Thus $N_{\Phi}\left(F_{m}-F^{\prime}\right) \leqslant 1 / K$ for $m \geqslant P_{K}$. The arbitrariness of $K$ implies $\varliminf_{\infty} N_{\Phi}\left(F_{m}-F\right)=0$, q.e.d.

As in the Orlicz spaces of point functions, a form of the Hölder inequality can be stated for the $A^{\Phi}(\mathscr{X})$-spaces.

LEMMA 12. If $\Phi$ and $\Psi$ are complementary Young's functions and $\mathscr{X}^{*}$ is the conjugate space of $\mathscr{X}$, then

$$
\sup _{\pi} \sum_{\pi} \frac{\left\|F\left(E_{n}\right)\right\|\left\|G\left(E_{n}\right)\right\|}{\mu\left(E_{n}\right)} \leqslant 2 N_{\Phi}\left(E^{\prime}\right) N_{Y^{\prime}}(G)
$$

for all $F \in A^{\Phi}(\mathscr{X})$ and $G \in A^{\Psi}\left(\mathscr{X}^{*}\right)$.

Proof. If either $F$ or $G$ is 0 , the result is immediate. Assuming neither $F$ nor $G$ is 0 , in view of Young's inequality (Proposition 2), one obtains

$$
\begin{aligned}
& \sum_{\pi} \frac{\frac{\left\|F\left(E_{n}\right)\right\|}{N_{\Phi}\left(F^{\prime}\right)} \frac{\left\|G\left(E_{n}\right)\right\|}{N_{\Psi}(G)}}{\mu\left(E_{n}\right)}=\sum_{\pi} \frac{\frac{\left\|F\left(E_{n}\right)\right\|}{N_{\Phi}\left(F^{\prime}\right)} \frac{\left\|G\left(E_{n}\right)\right\|}{N_{\Psi}(G)}}{\mu\left(E_{n}\right) \mu\left(E_{n}\right)} \mu\left(W_{n}\right) \\
& \leqslant \sum_{\pi} \Phi\left(\frac{\left\|F\left(E_{n}\right)\right\|}{N_{\Phi}\left(F^{\prime}\right) \mu\left(E_{n}\right)}\right) \mu\left(E_{n}\right)+\sum_{n} \Psi\left(\frac{\left\|G\left(E_{n}\right)\right\|}{N_{\Psi}(G) \mu\left(E_{n}\right)}\right) \mu\left(E_{n}\right) \\
& <I_{\Phi}\left(\frac{F}{N_{\Phi}\left(F^{\prime}\right)}\right)+I_{\Psi}\left(\frac{N}{N_{\Psi}(G)}\right) \leqslant 1+1=2
\end{aligned}
$$

for any partition $\pi$. Hence

$$
\sup _{\pi} \sum_{\pi} \frac{\frac{\left\|F^{\prime}\left(E_{n}\right)\right\|}{N_{\phi}\left(F^{\prime}\right)} \frac{\left\|G\left(E_{n}\right)\right\|}{N_{\Psi}(G)}}{\mu\left(E_{n}\right)} \leqslant 2,
$$

or,

$$
\sup _{\pi} \sum_{\pi} \frac{\left\|F^{\prime}\left(\mathbb{W}_{n}\right)\right\|\left\|G\left(\mathbb{W}_{n}\right)\right\|}{\mu\left(\mathbb{H}_{n}\right)} \leqslant 2 N_{\Phi}\left(F^{\prime}\right) N_{\mathrm{Tr}}(G), \quad \text { q. e. d. }
$$

If $\Phi(x)=|x|$, the resulting $A^{\Phi}(\mathscr{X})$-space is denoted by $A^{1}(\mathscr{X})$. Clearly the $A^{1}(\mathscr{X})$-norm is precisely that of the space of bounded $\mathscr{X}$-valued set functions, $b a(\Omega, \Sigma, \mathscr{X})$ - the variation norm $\mathscr{V}(\cdot)$. The next lemma establishes an interesting and useful relationship between $A^{\Phi}(\mathscr{X})$ and $A^{1}(\mathscr{X})$.

Lemma 13. If $E \in \Sigma_{0}$ and $F^{\prime} \in A^{\Phi}(\mathscr{X})$, then $F^{\cdot} \in \in A^{1}(\mathscr{X})$. Moreover, there exists a constant $K$ depending only on $\mu(E)$ such that

$$
\mathscr{V}\left(F^{\prime} \cdot E\right)=N_{1}(F \cdot E) \leqslant K N_{\Phi}(F) .
$$

In particular, if $\mu(\Omega)<\infty, A^{1}(\mathscr{X}) \subset A^{\Phi}(\mathscr{X})$ and there exists a constant $K$ such that $N_{1}\left(F^{\prime}\right) \leqslant K N_{\Phi}(F)$ for all $F \in A^{\Phi}(\mathscr{X})$.

Proof. From the support line property of convex functions, there exists a constant $K_{0}>0$ such that

$$
|x| \leqslant K_{0} \Phi(x)+K_{0} .
$$

Hence, if $0 \neq F \in A^{\Phi}(\mathscr{X})$ and $S \in \Sigma_{0}$ is arbitrary

$$
\frac{\left\|F^{\prime}(S)\right\|}{N_{\Phi}\left(F^{\prime}\right)} \leqslant K_{0} \Phi\left(\frac{\left\|F^{\prime}(S)\right\|}{\mu\left(S^{\prime}\right) N_{\Phi}\left(F^{\prime}\right)}\right) \mu(S)+K_{0} \mu(S)
$$

Accordingly, if $\pi=\left\{\mathbb{E}_{n}\right\}$ is a partition of $E$,

$$
\begin{aligned}
\frac{1}{N_{\Phi}\left(F^{\prime}\right)} \sum_{n}\left\|F^{\prime}\left(E_{n}\right)\right\| & \leqslant K_{0} \sum_{\pi} \Phi\left(\frac{\left\|F^{\prime}\left(E_{n}\right)\right\|}{\mu\left(E_{n}\right) N_{\Phi}\left(F^{\prime}\right)}\right) \mu\left(E_{n}\right)+K_{0} \sum_{n} \mu\left(E_{n}\right) \\
& \leqslant K_{0} I_{\Phi}\left(\frac{F}{N_{\Phi}\left(F^{\prime}\right)}\right)+K_{0} \mu(E)<\infty .
\end{aligned}
$$

Since $\pi$ is arbitrary, it follows that

$$
\frac{N_{1}(F \cdot E)}{N_{\Phi}(F)} \leqslant K_{0}[1+\mu(E)]=K<\infty .
$$


For a more detailed study, a subclass of the $A^{\Phi}(\mathscr{X})$-spaces having a more amenable structure will be isolated; the slightly more general case, not treated in the sequel, seems to require different methods of study.

Definition 14. $\nabla^{\Phi}(\Omega, \Sigma, \mu, \mathscr{X})\left(=V^{\Phi}(\mathscr{X})\right.$ stands for the linear submanifold of $A^{\Phi}(\mathscr{X})$ consisting of $\mu$-continuous functions (i.e.,

$$
\lim _{\mu(\mathbb{L}) \rightarrow 0}\|F(E)\|=0
$$

for each $F \in V^{\Phi}(\mathscr{X})$ ). $V^{\Phi}(\mathscr{X})$ stands for $V^{\Phi}(\mathscr{X}) \cap A^{\Phi}(\mathscr{X})$.

The following lemma due to Rao ([18], p. 85) shows that in many cases $A^{\Phi}(\mathscr{X})=V^{\Phi}(\mathscr{X})$ :

Lemra 15. If $\Phi$ and $\Psi$ are complementary Young's functions and $\Psi$ is continuous, then $A^{\Phi}(\mathscr{X})=V^{\Phi}(\mathscr{X})$.

The proof is given in [18], p. 85, for real-valued set functions defined on a $\sigma$-field. The proof there is applicable here mutatis mutandis.

In a more general setting is

THEOREM 16. $V^{\Phi}(\mathscr{X})$ is a closed subspace of $A^{\Phi}(\mathscr{X})$. Hence $V^{\Phi}(\mathscr{X})$ is a Banach space.

Proof. Clearly $V^{\Phi}(\mathscr{X})$ is a linear space. Therefore, all that need be shown is that $V^{\Phi}(\mathscr{X})$ is complete. Let $\left\{F_{n}\right\} \subset V^{\Phi}(\mathscr{X})$ be a Cauchy sequenco. The completeness of $A^{\Phi}(\mathscr{X})$ establishes the existence of $I^{\prime} \in A^{\Phi}(\mathscr{X})$ such that $\lim N_{\Phi}\left(F_{n}-F\right)=0$. According to lemma 15 , there exists a $K>0$ such that

$$
\mathscr{V}\left(F_{n} \cdot E-F \cdot E\right) \leqslant K N_{\Phi}\left(F_{n}-F^{\prime}\right)
$$

where $K$ is independent of $E \epsilon \Sigma_{0}$ provided $\mu(E) \leqslant 1$. Let $\varepsilon>0$ bo given and select $n_{0}$ such that

$$
N_{\Phi}\left(T_{n_{0}}-I^{\prime}\right)<\varepsilon / 2 K
$$

Then $\mathscr{V}\left(F_{n_{0}} \cdot E-F \cdot E\right)<\varepsilon / 2$. Since $F_{n_{0}}$ is $\mu$-continuous, there exists $\delta_{1}>0$ such that $\left\|F_{n_{0}}(E)\right\|<\varepsilon / 2$ whenever $\mu(t)<\delta_{1}$. Wherofore, if $\delta=\min \left\{\delta_{1}, 1\right\}$

$$
\mid\left\|F_{n_{0}}(E)\right\|-\|F(E)\| \leqslant \mathscr{V}\left(F_{n_{0}} \cdot E-T \cdot E\right)<\varepsilon / 2
$$

provided $\mu(E)<\delta$. It follows that $\|F(E)\|<\varepsilon$ whenever $\mu(E)<\delta$. Therefore $F \in V^{\Phi}(\mathscr{X})$ and $V^{\Phi}(\mathscr{X})$ is a closed. subspace of $A^{\Phi}(\mathscr{X})$, q. e. d.

Remark. If $\Phi(x)=|x|^{p}, \mathbf{1} \leqslant p \leqslant \infty, \mathscr{X}$ is the real line and $\mu(\Omega)$ is finite, the corresponding $V^{\infty}$-space is precisely the $V^{n}$-space introduced by Bochner [5,6] and Leader [13].
As in the theory of Orlicz spaces of point functions, the structure of $V^{\Phi}(\mathscr{X})$ cannot be completely analyzed without the introduction of a second norm. Corresponding to the Orlicz norm ([24], p. 79) on $L^{\Phi}(\mathscr{X})$ is the functional $\|\cdot\|_{\Phi}$ defined for $F$ in $V^{\Phi}(\mathscr{X})$ by

$$
\|F\|_{\Phi}=\sup \left\{\sup _{\pi} \sum_{\pi} \frac{\left\|F\left(E_{n}\right)\right\|\left\|G\left(E_{n}\right)\right\|}{\mu\left(E_{n}\right)} ; G \in V^{\Psi}\left(\mathscr{X}^{*}\right), N_{\Psi}(G) \leqslant 1\right\},
$$

where $\Psi$ is complementary to $\Phi$. Lemma 14 guarantees that

$$
\left\|F^{\prime}\right\|_{\Phi} \leqslant \sup _{N_{\Psi}(G) \leqslant 1} 2 N_{\Phi}\left(F^{\prime}\right) N_{\Psi}(G)=2 N_{\Phi}(F)
$$

so that $\|\cdot\|_{\Phi}$ is finite on $V^{\Phi}(\mathscr{X})$.

LEMMA 17. $\|\cdot\|_{\Phi}$ is a norm on $V^{\Phi}(\mathscr{X})$.

Proof. $\|F\|_{\infty}=0$ if and only if $F=0$. Clearly $F=0$ implies $\|F\|_{\Phi}=0$. If $F \in V^{\Phi}(\mathscr{X})$ and there exists $E \in \Sigma_{0}$ such that $F(E) \neq 0$, select $x^{*} \in \mathscr{X}^{*}$ such that $0<\Psi\left(\left\|x^{*}\right\|\right) \leqslant 1 / \mu(E)$ and let $H=x^{*} \mu \cdot E$. Then $N_{\Psi}(H) \leqslant 1$ and $\|F(E)\|\|H(E)\| / \mu(E) \geqslant 0$. Hence $\|F\|_{\Phi}>0$. That $\|\cdot\|_{\Phi}$ obeys the other norm properties follows immediately from the corresponding properties of the norm in $\mathscr{X}$, q. e. d.

The importance of $\|\cdot\|_{\Phi}$ will become apparent after it is shown that $\|\cdot\|_{\Phi}$ is actually equivalent to the $N_{\Phi}$-norm. This will follow directly from the following lemma:

LEMma 18. $I_{\Phi}\left(F /\|F\|_{\Phi}\right) \leqslant 1$ for all $F \neq 0$ in $V^{\Phi}(\mathscr{X})$.

Proof. Preliminary to the actual proof is the fact that for $F$ in $V^{\Phi}(\mathscr{X})$ and $G$ in $V^{p}\left(\mathscr{X}^{*}\right)$

$$
\sup _{\pi} \sum_{\pi} \frac{\left\|F\left(E_{n}\right)\right\|\left\|G\left(E_{n}\right)\right\|}{\mu\left(E_{n}\right)} \leqslant\left\{\begin{array}{lll}
\|F\|_{\Phi} & \text { if } & I_{\Psi}(G) \leqslant 1, \\
\|F\|_{\Phi} I_{\Psi}(G) & \text { if } & 1<I_{\Psi}(G)<\infty .
\end{array}\right.
$$

This follows as in the point function case ([24], p. 80) with essentially no modifications.

If $\Phi$ does not jump (i.e., $\Phi(x)<\infty$ for $x<\infty$ ), let $\pi=\left\{E_{n}\right\}$ be any partition and consider

$$
G=\sum_{\pi} x^{*} \varphi\left(\frac{\left\|F\left(E_{n}\right)\right\|}{\|F\|_{\Phi} \mu\left(E_{n}\right)}\right) \mu \cdot E_{n}
$$

where $x^{*}$ is an element of the unit sphere of $\mathscr{X}^{*}$. Since $G$ is a step function on $\pi$, and $\left\|x^{*}\right\|=1$, by theorem 9 ,

$$
I_{\Psi}(G)=\sum_{\pi} \Psi\left(\varphi\left(\frac{\left\|F\left(E_{v}\right)\right\|}{\|F\|_{\Phi} \mu\left(E_{n}\right)}\right)\right) \mu\left(E_{v}\right)
$$


With this choice of $G$,

$$
\begin{aligned}
& \sum_{\pi} \frac{\left\|F\left(E_{n}\right)\right\|\left\|G\left(E_{n}\right)\right\|}{\|F\|_{\Phi} \mu\left(E_{n}\right)}=\sum_{\pi} \frac{\left\|F\left(E_{n}\right)\right\|}{\|F\|_{\Phi} \mu\left(E_{n}\right)} \varphi\left(\frac{\left\|F\left(E_{n}\right)\right\|}{\|F\|_{\Phi} \mu\left(E_{n}\right)}\right) \mu\left(\mathbb{E}_{n}\right) \\
& =\sum_{n} \Phi\left(\frac{\left\|F\left(E_{n}\right)\right\|}{\|F\|_{\Phi} \mu\left(E_{n}\right)}\right) \mu\left(E_{n}\right)+\sum_{n} \Psi\left(p\left(\frac{\left\|\left(E_{n}\right)\right\|}{\left\|F^{\prime}\right\|_{\Phi} \mu\left(\mathbb{E}_{n}\right)}\right)\right) \mu\left(E_{n}\right),
\end{aligned}
$$

since with this $G$ there is term-by-term equality in Young's inequality,

$$
=\sum_{\pi} \Phi\left(\frac{\left\|F\left(E_{n}\right)\right\|}{\|F\|_{\Phi} \mu\left(E_{n}\right)}\right) \mu\left(E_{n}\right)+I_{w r}(G)
$$

Since the left-hand side is finite, so is the right-hand side and $I_{\Psi}(G)<\infty$. If $I_{I F}(G) \leqslant 1$, the preliminary result yields,

$$
\sum_{\pi} \Phi\left(\frac{\left\|F\left(E_{n}\right)\right\|}{\|F\|_{\Phi} \mu\left(E_{n}\right)}\right) \mu\left(E_{n}\right) \leqslant \sum_{\pi} \frac{\left\|F^{\prime}\left(E_{n}\right)\right\|\left\|G\left(E_{n}\right)\right\|}{\left\|F^{\prime}\right\|_{\Phi} \mu\left(E_{n}\right)} \leqslant 1 .
$$

If $I_{\Psi}(G)>1$, the same preliminary result implies

$$
I_{\Psi}(G) \geqslant \sum_{\pi} \frac{\left\|F\left(E_{n}\right)\right\|\left\|G\left(E_{n}\right)\right\|}{\|\|^{\prime} \mu\left(E_{n}\right)}=\sum_{\pi} \Phi\left(\frac{\left\|F^{\prime}\left(E_{n}\right)\right\|}{\left\|F^{\prime}\right\|_{\Phi} \mu\left(E_{n}\right)}\right) \mu\left(I_{n}\right)+I_{\Psi}(G)
$$

so that

$$
\sum_{\pi} \Phi\left(\frac{\left\|F^{\prime}\left(E_{n}\right)\right\|}{\|F\|_{\Phi} \mu\left(E_{n}\right)}\right) \mu\left(E_{n}\right)=0
$$

In either case

$$
\sum_{\pi} \Phi\left(\frac{\left\|F\left(E_{n}\right)\right\|}{\|F\|_{\Phi} \mu\left(E_{n}\right)}\right) \mu\left(E_{n}\right) \leqslant 1 .
$$

Since the partition $\pi$ is arbitrary

$$
I_{\Phi}\left(\frac{F}{\|F\|_{\Phi}}\right)=\sup _{\pi} \sum_{I I} \Phi\left(\frac{\left\|F\left(E_{n}\right)\right\|}{\|\vec{F}\|_{\Phi} \mu\left(E_{n}\right)}\right) \mu\left(\mathbb{E}_{n}\right) \leqslant 1 .
$$

This establishes the lemma if $\Phi$ is continuous.

Suppose $\Phi$ jumps at $M>0$. If it can be shown that

$$
\frac{\|F(E)\|}{\left\|F^{\prime}\right\|_{\Phi} \mu(E)} \leqslant M
$$

for any $E \in \Sigma_{0}$ of positive $\mu$-measure; then $\varphi\left(\|F(E)\| /\|F\|_{\Phi} \mu(E)\right)<\infty$, and the proof given above for continuous $\Phi$ applies without modification.
Since $\Phi$ jumps at $M, \Psi(x) \leqslant M$ for all $x$. Hence

$$
\Psi(x)=\int_{0}^{|x|} \Psi(t) d t \leqslant M|x|
$$

and $N_{\Psi}(H) \leqslant M N_{1}(H)$. For any $E \in \Sigma_{0}$ of positive $\mu$-measure, consider

$$
G=\frac{x^{*}}{M \mu(E)} \mu \cdot E,
$$

where $x^{*}$ is on the unit sphere of $\mathscr{X}^{*} . N_{\Psi}(G) \leqslant M N_{1}(G)=1$. Therefore

$$
\frac{\|F(E)\|\|G(E)\|}{\mu(E)} \leqslant\|F\|_{\Phi} .
$$

But

$$
\frac{\left\|F^{\prime}(E)\right\|\|G(E)\|}{\mu(E)}=\frac{\|F(E)\|}{\mu(E)} \cdot \frac{1}{M}
$$

Hence

$$
\frac{\left\|F^{\prime}(E)\right\|}{\| \boldsymbol{F}_{\Phi} \mu(E)} \leqslant M, \quad \text { q. e. d. }
$$

Some important consequences of this lemma are collected below:

THEOREM 19. (a) If $\Phi$ and $\Psi$ are complementary Young's functions, and $F \in V^{\Phi}(\mathscr{X}), G \in V^{\Psi}\left(\mathscr{X}^{*}\right)$, then the following Hölder type inequalities are valid:

$$
\sup _{\pi} \sum_{\pi} \frac{\left\|F\left(E_{n}\right)\right\|\left\|G\left(E_{n}\right)\right\|}{\mu\left(E_{n}\right)} \leqslant\left\{\begin{aligned}
\text { (i) } & \left\|F^{\prime}\right\|_{\Phi}\|G\|_{\Psi}, \\
\text { (ii) } & N_{\Phi}(F)\|G\|_{\Psi}, \\
\text { (iii) } & 2 N_{\Phi}\left(F^{\prime}\right) N_{\Psi}(G) .
\end{aligned}\right.
$$

(b) $N_{\Phi}(F) \leqslant\|F\|_{\Phi} \leqslant 2 N_{\Phi}(F)$.

Consequently $N_{\Phi}(\cdot)$ and \|\|$_{\Phi}$ are equivalent norms on $V^{\Phi}(\mathscr{X})$. In particular, $V^{\Phi}(\mathscr{X})$ is a Banach space under $\|\cdot\|_{\Phi}$ as well.

Proof. (a) If either $F$ or $G$ is 0 , the assertions (i), (ii), (iii), and (b) hold trivially. If neither $F$ nor $G$ is 0 , lemma 18 states $I_{\Psi}\left(G /\|G\|_{P}\right) \leqslant 1$. Hence $N_{\Psi}\left(G /\|G\|_{\Psi}\right) \leqslant 1$ and

$$
\sup _{\pi} \sum_{\pi} \frac{\left\|F\left(E_{n}\right)\right\|\left\|G\left(E_{n}\right)\right\|}{\mu\left(E_{n}\right)\|G\|_{\Psi}} \leqslant\|F\|_{\Phi}
$$

whence

$$
\sup _{\pi} \sum_{\pi} \frac{\left\|F\left(E_{n}\right)\right\|\left\|G\left(E_{n}\right)\right\|}{\mu\left(E_{n}\right)} \leqslant\|F\|_{\varnothing}\|G\|_{\varphi} .
$$

This establishes (i). 
To prove (ii), note that $N_{\Phi}\left(T / N_{\Phi}(F)\right) \leqslant 1$. Therefore,

$$
\sup _{\pi} \sum_{\pi} \frac{\left\|E^{\prime}\left(E_{n}\right)\right\|\left\|G\left(E_{n}\right)\right\|}{N_{\Phi}\left(F^{\prime}\right) \mu\left(E_{n}\right)} \leqslant\|G\|_{p}
$$

or

$$
\sup _{\pi} \sum_{n} \frac{\left\|F\left(E_{n}\right)\right\|\left\|G\left(E_{n}\right)\right\|}{\mu\left(E_{n}\right)} \leqslant N_{\Phi}(F)\|Q\|_{\varphi} .
$$

(iii) is already proved as lemma 12.

(b) It follows from (iii) that $\left\|F^{\prime}\right\|_{\Phi} \leqslant 2 N_{\Phi}\left(I^{\prime}\right)$. According to lemma 18 , $I_{\Phi}\left(F^{F} /\left\|H_{\Phi}\right\|\right) \leqslant 1$. The definition of $N_{\Phi}$ implies $N_{\Phi}\left(F^{\prime}\right) \leqslant\left\|I^{\prime}\right\|_{\Phi}$. Combining these inequalities, one obtains

$$
N_{\Phi}(F) \leqslant\left\|F^{\prime}\right\|_{\Phi} \leqslant 2 N_{\Phi}(F) .
$$

Consequently the two norms are equivalent, q. e. d.

\section{The Orliez spaces $L^{\Phi}(X)$ and the injection of $L^{\Phi}(X)$ into $V^{\phi}(X)$}

The basis for this section is also a non-negative extended real-valued finitely additive set function $\mu$ defined on a field $\Sigma$ of subsets of a set $\Omega$. $\Sigma_{0} \subset \Sigma$ is the ring of sets of finite $\mu$-measure. The (point) functions to be considered will be defined on $\Omega$, are totally $\mu$-measurable in the sense of [9] and have their values in a real or complex $B$-space $\mathscr{X}$. Orlicz spaces (possibly incomplete) of such functions, denoted by $L^{\Phi}(\mathscr{X})$ will be defined, and elementary properties of these spaces will be discpssed. Finally, an isometric injection of $L^{\Phi}(\mathscr{C})$ into the corresponding $V^{\Phi}(\mathscr{C})$-space will be constructed in a natural way. The integration procedure to be employed is that of [9], Chapter III, and coincides with the familiar Bochner integral when $\Sigma$ is a $\sigma$-field and $\mu$ is a countably additive measure.

Definition 1. Let $\Phi$ be a Young's function. $L_{0}^{\phi}(\Omega, \Sigma, \mu, \mathscr{X})$ $\left(=L_{0}^{\Phi}(\mathscr{C})\right)$ denotes the collection of all totally- $\mu$-measurable ([9], III 2.10) $\mathscr{X}$-valued functions $f$ such that

$$
\int_{\Omega} \Phi\left(\frac{\|f\|}{k}\right) d \mu \leqslant 1 \quad \text { for some } l<>0 .
$$

By the norm $N_{\Phi}(f)$ of an element $f \in L_{0}^{\Phi}(\mathscr{X})$ is meant the quantity

$$
N_{\Phi}(f)=\inf \left\{k>0: \int_{\Omega} \Phi\left(\frac{\|f\|}{l_{i}}\right) d \mu \leqslant 1\right\} .
$$

Lemma 2. $L_{0}^{\Phi}(\mathscr{X})$ is a semi-normed linear space under the semi-norm $N_{\Phi}(\cdot)$. Moreover $N_{\Phi}(f)=0$ if and only if $f$ is $\mu$-null.

Proof. The second statement follows from standard properties of the integral. The proof of the fact that $N_{\phi}(\cdot)$ is a semi-norm is essentially the same as the proof of theorem I. 11 and need not be repeated here.

In view of lemma 2 , it is natural to consider classes of functions in $L_{0}^{\Phi}(\mathscr{X})$ equivalent under the relation: $f$ is equivalent to $g$ if and only if $f-g$ is a $\mu$-null function. With this identification, we denote the resulting space by $L^{\Phi}(\Omega, \Sigma, \mu, \mathscr{X})\left(=L^{\Phi}(\mathscr{X})\right)$. The following theorem is a direct consequence of lemma 2 :

THEOREM 3. $L^{\Phi}(\mathscr{X})$ is a normed linear space.

Unfortunately $L^{\mathscr{\Phi}}(\mathscr{X})$ is not, in general, a complete space. If it is insisted that $\Sigma$ be a $\sigma$-field and $\mu$ be countably additive, then the $L^{\Phi}(\mathscr{X})$ spaces defined above are automatically complete, and in fact, coincide with the familiar Orlicz spaces $[11,22,24]$ when $\mathscr{X}=R$, the real line. For this reason, it is natural to call $L^{\Phi}(\mathscr{X})$ an Orlicz space. Hereafter a function $f \epsilon L^{\Phi}(\mathscr{X})$ means that $f$ is a representative of its equivalence class. Paralleling ([22], Th. 6) is the following

THEOREM 4. If $f$ is in $L^{\Phi}(\mathscr{X})$ and $g$ is in $L^{\Psi}\left(\mathscr{X}^{*}\right)$, where $\Phi$ and $\Psi$ are complementary in the sense of Young, then $\|f\|\|g\|$ is integrable and

$$
\int_{\Omega}\|f\|\|g\| d \mu \leqslant 2 N_{\Phi}(f) N_{\Psi}(g)
$$

The proof is essentially the same as that of [22], Th. 6 , and will be omitted.

The next result links the $L^{\Phi}(\mathscr{X})$ and the $V^{\Phi}(\mathscr{X})$-spaces and allows many properties of $L^{\Phi}(\mathscr{X})$ to be deduced from the corresponding properties of $V^{\Phi}(\mathscr{C})$.

THEOREM 5. Let $\Phi$ be continuous.

(a) Each $f \in L^{\Phi}(\mathscr{X})$ is integrable on all sets of finite $\mu$-measure. Consequently, the set function $\lambda f$ defined on $\Sigma_{0}$ by

$$
\lambda f(E)=\int_{E} f d \mu, \quad E \epsilon \Sigma_{0},
$$

is finitely additive and $\mu$-continuous.

(b) If $f \in L^{\Phi}(\Omega, \Sigma, \mu, \mathscr{X})$, then $\lambda f \in V^{\Phi}(\Omega, \Sigma, \mu, \mathscr{C})$. The mapping $\lambda$ : $L^{\Phi}(\mathscr{X}) \rightarrow V^{\Phi}(\mathscr{X})$ is linear and $N_{\Phi}(f)=N_{\Phi}(\lambda f)$. Hence $\lambda$ is an isometric injection of $L^{\Phi}(\mathscr{X})$ into $V^{\Phi}(\mathscr{X})$.

Proof. The proof of the fact that $f \epsilon L^{\Phi}(\mathscr{X})$ is integrable on all sets of finite measure is based on the support line property of convex functions. Its proof is the same as the proof of lemma I. 13 with an obvious notational Studia Mathematica XXIX.1 
modification. The finite additivity of the set function $\lambda f$ follows from standard properties of the integral. Again from the support line property of $\Phi$, for $0 \neq f \in L^{\Phi}(\mathscr{X})$, one has

$$
\frac{\|\lambda f(E)\|}{N_{\Phi}(f)} \leqslant \int_{E} \frac{\|f\| d \mu}{N_{\Phi}(f)} \leqslant K \int_{E} \Phi\left(\frac{\|f\|}{N_{\Phi}(f)}\right) d \mu+K \mu(E),
$$

for some $K>0$, and all $H \in \Sigma_{0}$. The $\mu$-continuity of the right-hand side therefore implies the $\mu$-continuity of the left-hand side.

(b) To prove $\lambda f \in V^{\Phi}(\mathscr{X})$, it will be shown that $\|\lambda f\|_{\Phi}<\infty$. Let $G \in V^{W}\left(\mathscr{X}^{*}\right), N_{\Psi^{\prime}}(G) \leqslant 1$ and the partition $\pi$ be arbitrary. Then

$$
\begin{aligned}
& \sum_{\pi} \frac{\left\|\lambda f\left(E_{n}\right)\right\|\left\|G\left(E_{n}\right)\right\|}{\mu\left(E_{n}\right)}=\sum_{\pi} \frac{\left\|E_{E_{n}} f d \mu\right\|\left\|G\left(E_{n}\right)\right\|}{\mu\left(E_{n}\right)} \\
& \leqslant \sum_{\pi} \int_{E_{n}}\|f\| d \mu \frac{\left\|G\left(E_{n}\right)\right\|}{\mu\left(E_{n}\right)}=\int_{\Omega}\|f\|\left\|g_{\pi}\right\| d \mu, \quad y_{\pi}=\sum_{\pi} \frac{G\left(E_{n}\right)}{\mu\left(E_{n}\right)} \chi_{E_{n}},
\end{aligned}
$$

but

$$
\int_{\Omega} \Psi\left(\left\|g_{\pi}\right\|\right) d \mu=I_{\Psi}\left(G_{\pi}\right) \leqslant I_{\Psi}\left(G^{*}\right)
$$

by theorem I. 9 , where $\Psi$ is complementary to $\Phi$. Since $N_{\Psi^{\prime}}(G) \leqslant 1$, $I_{\Psi}(G) \leqslant 1$ and $N_{\Psi}\left(g_{\pi}\right) \leqslant 1$. An application of theorem 4 yields

$$
\sum_{\pi} \frac{\left\|\lambda f\left(E_{n}\right)\right\|\left\|G\left(\mathbb{E}_{n}\right)\right\|}{\mu\left(E_{n}\right)} \leqslant \int_{\Omega}\|f\|\left\|g_{\pi}\right\| d \mu \leqslant 2 N_{\Phi}(f) N_{\Psi}\left(g_{\pi}\right) \leqslant 2 N_{\Phi}(f) .
$$

Since $\pi$ and $G$ were arbitrary,

$$
\|\lambda f\|_{\Phi}=\sup _{N_{\Psi}(G) \leqslant 1} \sup _{\pi} \sum_{\pi} \frac{\left\|\lambda f\left(E_{n}\right)\right\|\left\|G\left(E_{v}\right)\right\|}{\mu\left(E_{n}\right)} \leqslant 2 N_{\Phi}(f) .
$$

Therefore $\|\lambda f\|_{\Phi}<\infty$. According to lemma I. 18, $I_{\Phi}\left(\lambda f /\|\lambda f\|_{\Phi}\right) \leqslant 1$. Hence $f \in V^{\Phi}(\mathscr{X})$.

To prove $N_{\Phi}(f)=N_{\Phi}(\lambda f)$, it suffices to show

$$
\Phi\left(\frac{\|f\|}{h^{\prime}}\right) d \mu=I_{\Phi}\left(\frac{\lambda f}{k}\right)
$$

for any $k>0$ such that $I_{\Phi}(\lambda f / k) \leqslant 1$. (To complete the proof a few results from section IV concerning the behavior of $I_{\phi}(\cdot)$ are needed. Since these results are independent of the $L^{\Phi}(\mathscr{X})$ theory, they will be used here.)
Let $k$ be such that $I_{\Phi}(\lambda f / k) \leqslant 1$ and $E \epsilon \Sigma_{0}$ be arbitrary. For each partition $\pi$ of $E$, consider the $\mu$-simple function

$$
\frac{f_{\pi}}{k}=\sum_{\pi} \frac{\int_{n} f d \mu}{\mu\left(E_{n}\right)} \chi_{E_{n}}
$$

From (a), $f \chi_{E} / k \in L^{1}(\mathscr{X})$. The proof of [9], IV. 8.17, shows that

$$
\lim _{\pi} \int_{E} \frac{\left\|f-f_{\pi}\right\|}{k} d \mu=0
$$

where the limit is taken through all partitions $\pi$ of $E$. It follows that the net $\left\{f_{\pi}\right\}$ converges in $\mu$-measure to $f \chi_{E}$. By virtue of the continuity of $\Phi$ and [9], III. 2.12, the net $\Phi\left(\left\|f_{\pi}\right\| / k\right)$ converges to $\Phi(\|f\| / k) \chi_{E}$ in $\mu$-measure. Now, for each $E_{0} \in \Sigma_{0}$ contained in $E$,

$$
\text { (A) } \int_{E_{0}} \Phi\left(\frac{\left\|f_{n}\right\|}{k}\right) d \mu=\sum_{\pi} \Phi\left(\frac{\left\|\int_{E_{n}} f d \mu\right\|}{k \mu\left(E_{n}\right)}\right) \mu\left(E_{v} \frown E_{0}\right)=I_{\Phi}\left(\left(\frac{\lambda f}{k}\right)_{\pi}, E_{0}\right)
$$

for each partition $\pi$ of $E$. Since $\lambda\left(f \chi_{E}\right)$ is an indefinite integral, the hypotheses of lemma IV. 5 (b) are satisfied and

$$
I_{\Phi}(\lambda f, E \cap \cdot)=\lim _{\pi} I_{\Phi}\left(\lambda f_{\pi}, E \cap \cdot\right)
$$

strongly in $V^{1}(R)$. This fact and [7], III. 2.15, imply

$$
\lim _{\pi, \Delta} \int_{E}\left|\Phi\left(\frac{\left\|f_{\pi}\right\|}{k}\right)-\Phi\left(\frac{\left\|f_{\Delta}\right\|}{k}\right)\right| d \mu=0,
$$

where the limit is taken through all partitions $\pi$ and $\Delta$ of $E$. Hence the net $\left\{\Phi\left(\left\|f_{\pi}\right\| / k\right), \pi\right.$ a partition of $\left.E\right\}$ determines $\Phi(\|f\| / k) \chi_{E}$ in the sense of [9], Chapter III; i.e.

$$
\int_{E} \Phi\left(\frac{\|f\|}{\hbar}\right) d \mu=\lim _{\pi} \int_{E} \Phi\left(\frac{\left\|f_{\pi}\right\|}{\hbar}\right) d \mu
$$

On the other hand, (B) implies

$$
I_{\Phi}\left(\frac{\lambda f}{k}, E\right)=\lim _{\pi} I_{\Phi}\left(\frac{f_{\pi}}{k}, E\right)=\lim _{\pi} \int_{E} \Phi\left(\frac{\left\|f_{\pi}\right\|}{k}\right) d \mu
$$

by (A),

$$
I_{\Phi}\left(\frac{\lambda f}{l_{c}}, E\right)=\int_{E} \Phi\left(\frac{\|f\|}{k}\right) d \mu
$$


from above. Hence

$$
I_{\Phi}\left(\frac{\lambda f}{k}, E\right)=\int_{E} \Phi\left(\frac{\|f\|}{k}\right) d \mu
$$

for each $E \in \Sigma_{0}$. From the definitions of $I_{\Phi}(\lambda f / k)$ and $\int_{\Omega} \Phi(\|f\| / k) d \mu$, it follows that

$$
\begin{aligned}
\int_{\Omega} \Phi\left(\frac{\|f\|}{k}\right) d \mu & =\sup _{E \in \Sigma_{0}} \int_{E} \Phi\left(\frac{\|f\|}{\lambda}\right) d \mu \\
& =\sup _{E \in \Sigma_{0}} I_{\Phi}\left(\frac{\lambda f}{k}, E\right), \quad \text { from above, } \\
& =I_{\Phi}\left(\frac{\lambda f}{k}, \Omega\right)=I_{\Phi}\left(\frac{\lambda f}{k}\right), \quad \text { q. ө. d. }
\end{aligned}
$$

Remark. In general the mapping $\lambda$ is not a surjection. Indeed, if $\lambda$ were always onto $V^{\Phi}(\mathscr{X})$, this would imply that each member of $V^{\Phi}(\mathscr{X})$ had a Radon-Nikodym derivative. This is not true in general - even when $\Sigma$ is a $\sigma$-field and $\mu$ is a countably additive finite measure on $\Sigma$. In fact, a counterexample may be found, for instance, in [23], p. 135. In addition, the image of $L^{\Phi}(\mathscr{X})$ under $\lambda$ may fail to be a closed subspace of $V^{\Phi}(\mathscr{X})$. Since $\lambda$ is an isometry, this will be true if and only if $L^{\Phi}(\mathscr{X})$ is complete.

Recall that $\mu$ has the finite subset property (FSP) if and only if for every set $E \in \Sigma$ of infinite measure, there exists $E_{0} \in \Sigma_{0}, E_{0} \subset E$ such that $0<\mu\left(E_{0}\right)<\mu(E)$. When $\mu$ has FSP, the classical Orlicz norm may be introduced on the $L^{\Phi}(\mathscr{X})$-spaces.

THEOREM 6 . Let $\Phi$ be continuous and $\mu$ prove the finite subset property. Then

(a) the functional $\|\cdot\|_{\Phi}$ defined for $f \epsilon L^{\Phi}(\mathscr{X})$ by

$$
\|f\|_{\Phi}=\sup \left\{\int_{\Omega}\|f\|\|g\| d \mu: g \in L^{\mid x}\left(\mathscr{X}^{*}\right), N_{\Psi r}(g) \leqslant 1\right\},
$$

where $\Psi$ is complementary to $\Phi$, is a norm.

(b) If $f \in L^{\Phi}(\mathscr{X})$, then $N_{\Phi}(f) \leqslant\|f\|_{\Phi} \leqslant 2 N_{\Phi}(f)$. Consequently, $N_{\Phi}(\cdot)$ and $\|\cdot\|_{\Phi}$ are equivalent norms on $L^{\Phi}(\mathscr{X})$.

(c) $\|\lambda f\|_{\Phi}=\|f\|_{\Phi}$ for all $f \in M^{\Phi}(\mathscr{X})$, where $M^{\Phi}(\mathscr{X})$ is the olosed subspace of $L^{\Phi}(\mathscr{X})$ determined by $\mu$-simple functions, and $\lambda$ is the injection of theorem 5 .

Proof. (a) From theorem 4, it immediately follows that $\|\cdot\|_{\phi}$ is well defined and finite on $L^{\Phi}(\mathscr{X})$. Arguments paralleling those of [24], p. 78-83, show that $\|\cdot\|_{\infty}$ is positive homogeneous and obeys the triangle inequality. Furthermore, it is clear that $\|f\|_{\Phi}=0$ if $f$ is $\mu$-null. The FSP will be used to prove the converse of this statement. If $f$ is not $\mu$-null, there exists by the proof of [7], III. 2.22 , a sequence of $\mu$-simple functions converging to $f$ in $\mu$-measure and satisfying $\left\|f_{n}\right\| \leqslant 2\|f\|$. It follows that there exists a set $E \in \Sigma$ and a positive number $a$ such that $\left\|f \chi_{E}\right\| \geqslant a \chi_{E}$. By FSP, there exists $E_{0} \epsilon \Sigma_{0}, E_{0} \subset E$ and $0<\mu\left(E_{0}\right)$. For this $E_{0}$, one has $\left\|f \chi_{E_{0}}\right\| \geqslant a \chi_{E_{0}}$. If $0 \neq x^{*} \epsilon \mathscr{X}^{*}$ is chosen such that $\Psi\left(\left\|x^{*}\right\|\right) \mu\left(E_{0}\right) \leqslant 1$ and $g=x^{*} \chi_{E}$, then $N_{\Psi}(g) \leqslant 1$ and

$$
\int_{\Omega}\|f\|\|g\| d \mu>0
$$

Hence $\|f\|_{\Phi}>0$. This proves (a)

(b) Let $f \in L^{\Phi}(\mathscr{X})$ be arbitrary. It will be shown first that $\|\lambda f\|_{\Phi} \leqslant\|f\|_{\Phi}$. Let $\varepsilon>0$ be given; choose $G \epsilon V^{\Psi^{\prime}}\left(\mathscr{X}^{*}\right), N_{\Psi}(G) \leqslant 1$ and the partition $\pi$ such that

$$
\sum_{\pi} \frac{\left\|\lambda f\left(E_{n}\right)\right\|\left\|G\left(E_{n}\right)\right\|}{\mu\left(E_{n}\right)} \geqslant\|\lambda f\|_{\Phi}-\varepsilon .
$$

Let

$$
g=\sum_{x} \frac{G\left(E_{n}\right)}{\mu\left(E_{n}\right)} \chi_{E_{n}} .
$$

Then $G_{\pi}=\lambda g$ and $N_{\Psi}(g)=N_{\Psi}\left(G_{\pi}\right) \leqslant N_{\Psi}(G) \leqslant 1$ by theorem $5(\mathrm{~b})$ and the choice of $G$. With this $g$, one has

$$
\begin{aligned}
\|f\|_{\Phi} & \geqslant \int_{\Omega}\|f\|\|g\| d \mu=\sum_{\pi} \frac{\int_{E_{n}}\|f\| d \mu\left\|G\left(E_{n}\right)\right\|}{\mu\left(E_{n}\right)} \\
& \geqslant \sum_{\pi} \frac{\left\|\int_{E_{n}} f d \mu\right\|\left\|G\left(E_{n}\right)\right\|}{\mu\left(E_{n}\right)}=\sum_{\pi} \frac{\left\|\lambda f\left(E_{n}\right)\right\|\left\|G\left(E_{n}\right)\right\|}{\mu\left(E_{n}\right)} \geqslant\|\lambda f\|_{\Phi}-\varepsilon
\end{aligned}
$$

by the choice of $G$.

Since $\varepsilon>0$ is arbitrary, it follows that $\|f\|_{\Phi} \geqslant\|\lambda f\|_{\Phi}$. But

$$
\|\lambda f\|_{\Phi} \geqslant N_{\Phi}(\lambda f)=N_{\Phi}(f)
$$

by theorem I. 19 (b) and theorem 5. On the other hand, from the definition of $\|f\|_{\Phi}$ and theorem 4, one has $\|f\|_{\Phi} \leqslant 2 N^{\Phi}(f)$. Combining these inequalities yields

and (b) follows.

$$
N_{\Phi}(f) \leqslant\|f\|_{\Phi} \leqslant 2 N_{\Phi}(f),
$$

(c) It is sufficient to prove $\|f\|_{\Phi}=\|\lambda f\|_{\Phi}$ for all $\mu$-simple functions $f \in L^{\Phi}(\mathscr{X})$. From the proof of $(\mathrm{b}),\|\lambda f\|_{\Phi} \leqslant\|f\|_{\Phi}$; thus it remains only to prove the opposite inequality. Let

$$
f=\sum_{i=1}^{n} x_{i} \chi_{E_{i}}, \quad x_{i} \in \mathscr{X}, \quad\left\{E_{i}\right\}_{i=1}^{n} \subset \Sigma_{0}
$$


be disjoint. If $g \in L^{\Psi}\left(\mathscr{X}^{*}\right), N_{\Psi}(g) \leqslant 1$, then

$$
\int_{\Omega}\|f\|\|g\| d \mu=\sum_{i=1}^{n}\left\|x_{i}\right\| \int_{E_{i}}\|g\| d \mu=\sum_{\pi} \frac{\left\|\lambda f\left(E_{i}\right)\right\| \int_{E_{i}}\|g\| d \mu}{\mu\left(E_{i}\right)},
$$

where $\pi$ is the partition $\left\{E_{i}\right\}_{i=1}^{n}$. Let $x^{*} \in \mathscr{X}^{*}$ be on the unit sphere and define $G$ on $\Sigma_{0}$ by

$$
G(E)=\int_{I} x^{*}\|g\| d \mu
$$

By the choice of $x^{*}$ and theorem $5(b)$,

Moreover

$$
N_{\Psi}(G)=N_{\Psi}\left(x^{*}\|g\|\right)=N_{Y}(g) \leqslant 1 .
$$

$$
\sum_{\pi} \frac{\left\|\lambda f\left(E_{i}\right)\right\| \int_{\mathbb{E}_{i}}\|g\| d \mu}{\mu\left(E_{i}\right)}=\sum_{\pi} \frac{\left\|\lambda f\left(\mathbb{E}_{i}\right)\right\|\left\|G\left(\mathbb{E}_{i}\right)\right\|}{\mu\left(E_{i}\right)} .
$$

It follows from this that $\|f\|_{\Phi} \leqslant\|\lambda f\|_{\Phi}$, q. e.d.

\section{A generalization of the Radon-Nikodym-Bochner theorem}

In this section, we shall investigate some special properties of vectorvalued set functions. A generalization of the Radon-Nikodym-Bochner theorem ([9], IV. 9. 14) for vector-valued finitely additive set functions is proved. This result is employed in this paper crucially in the study of the structure of $V^{\Phi}(\mathscr{X})$. By ba $(\Omega, \Sigma, \mathscr{X})$ is meant, as usual, the collection of all finitely additive $\mathscr{X}$-valued set functions of bounded variation defined on a field $\Sigma$ of subsets of $\Omega$, where $\mathscr{C}$ is a real or complex $B$-space. ca $(\Omega, \Sigma, \mathscr{X})$ is the collection of all countably additive members of ba $(\Omega, \Sigma, \mathscr{X}) . \Omega$ and $\Sigma$ will be fixed throughout this section. The following lemmas are extracted from [9], IV. 9, for ready reference.

LEMMa 1. There exists a totally disconnected Hausdorff space $S_{1}$ and an isomorphism $\tau: ' \Sigma \rightarrow \Sigma_{1}$, the field of all open and closed subsets of $S_{1}$ in the sense that $\tau\left(\mathbb{E} \cup F^{\prime}\right)=\tau(E) \cup \tau(F), \tau\left(E \cap F^{\prime}\right)=\tau(E) \cap \tau\left(F^{\prime}\right)$, and $\tau\left(E^{\prime}\right)=\tau(E)^{\prime}\left(E^{\prime}=\Omega-E\right)$ for all $E, E^{\prime} \in \Sigma$.

LEMMA 2. (a) If $C$ is the complex plane, there is an isometric isomorphism $T$ of ba $(\Omega, \Sigma, C)$ onto ba $\left(S_{1}, \Sigma_{1}, C\right)$ determined by the correspondence $T \mu\left(E_{1}\right)=\mu\left(\tau^{-1}\left(E_{1}\right)\right)$ for $\mu \in$ ba $\left(S_{1}, \Sigma_{1}, C\right)$ and $E_{1} \in \Sigma_{1}$.

(b) Each $\mu_{1}$ in ba $\left(S_{1}, \Sigma_{1}, C\right)$ has a unique extension to a regular countably additive measure $\mu_{2}$ in ca $\left(S_{1}, \Sigma_{2}, C\right)$ where $\Sigma_{2}$ is the o-field generated by $\Sigma_{1}$. The correspondence $U: \mu_{1} \rightarrow \mu_{2}$ is an isometrio isomorphism of ba $\left(S_{1}, \Sigma_{1}, C\right)$ onto ca $\left(S_{1}, \Sigma_{2}, C\right)$.
The following theorem generalizes the Radon-Nikodym-Bochner theorem ([9], IV. 9. 14) to vector-valued set functions and constitutes the main result of this section:

THEOREM 3. Let $\mathscr{X}$ be a Banach space, $\mu$ be a non-negative element of ba $(\Omega, \Sigma, C)$ and $F \in \mathrm{ba}(\Omega, \Sigma, \mathscr{X})$ be $\mu$-continuous. If

$$
\left\{\frac{F(E)}{\mu(E)}:\left\|\frac{F(E)}{\mu(E)}\right\| \leqslant n, \quad E \epsilon \Sigma\right\}
$$

is weakly sequentially compact in $\mathscr{X}$ for each positive integer $n$, then for each $\varepsilon>0$ there exists a $\mu$-simple function $f_{\varepsilon}$ such that $\mathscr{V}\left(F(\cdot)-\int_{(\cdot)} f_{\varepsilon} d \mu\right)<\varepsilon$, where $\mathscr{V}$ is the variation norm on ba $(\Omega, \Sigma, \mathscr{X})$.

Proof. The notation of lemmas 1 and 2 will be used throughout the proof. In the notation of lemma 2 , let $M=U T$; by lemma $2, M$ is an isometric isomorphism of ba $(\Omega, \Sigma, C)$ onto ca $\left(S_{1}, \Sigma_{2}, C\right)$. Let $M(\mu)$ $=\mu_{2}$, and define $F_{1}$ on $\Sigma_{1}$ by $F_{1}\left(E_{1}\right)=F\left(\tau^{-1}\left(E_{1}\right)\right)$ for $E_{1} \in \Sigma_{1}$. Since $F$ is $\mu$-continuous, it follows that $F_{1}$ is $\mu_{2}$-continuous on $\Sigma_{1}$. Next $F_{1}$ will be extended to $\Sigma_{2}$, the $\sigma$-field generated by the field $\Sigma_{1}$. According to [9], III. 7.1, for each $E$ in $\Sigma_{2}$, there exists a sequence $\left\{E_{n}\right\}$ in $\Sigma_{1}$ such that $\mu_{2}-\lim E_{n}=E$ in the sense that $\lim \mu_{2}\left(E \Delta E_{n}\right)=0$, where $\Delta$ is the symmetric difference operation. Now, if $\mathbb{H}_{\epsilon} \in \Sigma_{2}$ is arbitrary and $\left\{E_{n}\right\} \subset \Sigma_{1}$ satisfies $\mu_{2}-\lim E_{n}=E$, then from the $\mu_{2}$-continuity of $F_{1}$ on $\Sigma_{1}$ and the simple relations $\mu_{2}\left(E_{m} \Delta E_{n}\right)=\mu_{2}\left(E_{m}-E_{m} \cap E_{n}\right)+\mu_{2}\left(E_{n}-E_{m} \cap E_{n}\right)$ and $\left\|F_{1}\left(E_{m}\right)-F_{1}\left(E_{n}\right)\right\|=\left\|F_{1}\left(E_{m}-E_{m} \frown E_{n}\right)-F_{2}\left(E_{n}-E_{m} \frown E_{n}\right)\right\| \leqslant \| F_{1}\left(E_{m}-\right.$ $\left.-E_{m} \cap E_{n}\right)\|+\| F^{\prime}\left(E_{n}-E_{n} \cap E_{m}\right) \|$, it follows that $\lim _{n, m}\left\|F_{1}\left(E_{n}\right)-F_{1}\left(E_{m}\right)\right\|$ $=0$. The completeness of $\mathscr{X}$ insures the existence of an element $x_{E}$ in $\mathscr{X}$ such that $\lim \mathbb{F}_{1}\left(E_{n}\right)=x_{E}$ strongly in $\mathscr{X}$. It is clear that $x_{E}$ is independent of the sequence $\left\{E_{n}\right\}$ converging to $E$ since any two sequences converging to $E$ can be combined into a single sequence converging to $E$. Define the function $F_{2}$ on $\Sigma_{2}$ by $F_{3}\left(E_{1}\right)=F_{1}\left(E_{1}\right)$ for all $E_{1}$ in $\Sigma_{1}$ and by $F_{2}(E)=x_{E}$ if $E$ is not in $\Sigma_{1}$ but is in $\Sigma_{2}$.

The next step is to show that the thus defined $F_{2}$ is strongly countably additive, $\mu_{2}$-continuous, and of bounded variation. Let $x^{*} \in \mathscr{X}^{*}$ be arbitrary. By lemma $2, x^{*} F_{1}$ has a unique countably additive extension, say $G_{x}^{*}$ to $\Sigma_{2}$. On account of the fact that $F_{1}$ is $\mu_{2}$-continuous on $\Sigma_{1}, x^{*} F_{1}$ is $\mu_{2}$-continuous on $\Sigma_{1}$. An application of [9], IV. 9. 13, implies $G_{x}^{*}$ is $\mu_{2}$-continuous. Replacing $G_{x}^{*}$ in the argument used to define $F_{2}$, one finds $\lim x^{*}\left(F_{1}\left(E_{n}\right)\right)=G_{x}^{*}(E)$ for any sequence $\left\{E_{n}\right\}$ in $\Sigma_{1}$ such that $\mu_{2}-\lim E_{n}{ }^{n}=E$ in $\Sigma_{2}-\Sigma_{1}$. Since strong convergence implies weak convergence, it follows that $G_{x}^{*}(E)=x^{*}\left(F_{2}(E)\right)$ for all $E \in \Sigma_{2}$. But $G_{x}^{*}$ is 
countably additive and $x^{*} \epsilon \mathscr{P}^{*}$ is arbitrary. Therefore $F_{2}$ is weakly countably additive. An application of a result of Pettis ([9], IV. 10.1) shows that $F_{2}$ is strongly countably additive on $\Sigma_{2}$. To show $F_{2}$ is $\mu_{2}$-continuous, note that $G_{x}^{*}\left(=x^{*} F_{2}\right)$ is $\mu_{2}$-continuous for each $x^{*} \epsilon \mathscr{C}^{*}$. Therefore $x^{*} F_{2}(N)$ $=0$ for all $x^{*} \in \mathscr{Q}^{*}$ whenever $N \epsilon \Sigma_{2}$ and $\mu_{2}(N)=0$. Hence $F_{2}$ vanishes on $\mu_{2}$-null sets. Another theorem of Pettis ([9], IV. 10.1) then implies $F_{2}$ is $\mu_{2}$-continuous.

Next, it will be shown that $\mathscr{V}\left(F_{2}\right)=\mathscr{V}\left(F^{\prime}\right)$. For, $\mathscr{V}\left(F^{\prime}\right) \leqslant \mathscr{V}\left(H_{2}\right)$ since $F$ is "defined" only on a subclass (under identification) of $\Sigma_{2}$. From [13], Th. 9,

$$
\begin{aligned}
0 & =\lim _{\pi} \mathscr{V}\left(x^{*} F_{\pi}-x^{*} F^{\prime}\right)=\lim _{\pi} \mathscr{V}\left(M\left(x^{*} F_{\pi}-x^{*} F^{\prime}\right)\right) \\
& =\lim _{\pi} \mathscr{V}\left(M\left(x^{*} F_{\pi}\right)^{\prime} M\left(x^{*} F^{\prime}\right)\right),
\end{aligned}
$$

since $M$ is an isometry. Therefore

$$
\lim _{\pi} M\left(x^{*} F_{\pi}\right)\left(E_{2}\right)=M\left(x^{*} F\right)\left(E_{2}\right)
$$

for all $E_{2} \epsilon \Sigma_{2}$ and $x^{*} \in \mathscr{C}^{*}$. But $M\left(x^{*} F^{*}\right)\left(H_{1}\right)=x^{*} F_{1}\left(W_{1}\right)$ for all $E_{1} \in \Sigma_{1}$. Lemma 2 (b) implies, by the uniqueness of the extension of $x^{*} F_{1}$ to $\Sigma_{2}$, that $M\left(x^{*} F^{\prime}\right)\left(E_{2}\right)=x^{*} F_{2}\left(E_{2}\right)$ for all $E_{2} \in \Sigma_{2}$. Now, if $\pi$ is any partition of $\Omega$, and $x^{*} \in \mathscr{X}^{*}$

$$
\begin{aligned}
M\left(x^{*} F_{\pi}\right) & =M\left(\sum_{\pi} \frac{x^{*} F\left(E_{n}\right)}{\mu\left(E_{n}\right)} \mu \cdot E_{n}\right)=\sum_{\pi} \frac{x^{*} F^{\prime}\left(E_{n}\right)}{\mu\left(E_{n}\right)} M\left(\mu \cdot E_{n}\right) \\
& =\sum_{\pi} \frac{x^{*} F\left(E_{n}\right)}{\mu\left(E_{n}\right)} \mu_{2} \cdot \tau\left(E_{n}\right) \\
& =\sum_{\pi} \frac{x^{*} F_{2}\left(\tau\left(E_{n}\right)\right)}{\mu_{2}\left(\tau\left(E_{n}\right)\right)} \mu_{2} \cdot \tau\left(E_{n}\right)=x^{*} F_{2_{\tau(\pi)}}
\end{aligned}
$$

where $\tau(\pi)=\left\{\tau\left(E_{n}\right): E_{n} \epsilon \pi\right\}$. Combining these results, one obtains

$$
\lim _{\pi} x^{*} F_{2_{\tau(\pi)}}(E)=x^{*} F_{2}(E)
$$

for all $E \in \Sigma_{2}$ and $x^{*} \in X^{*}$. It follows that

$$
\left\|F_{2}(E)\right\| \leqslant \underset{\pi}{\liminf }\left\|F_{2 \tau(\pi)}(E)\right\|
$$

for all $E \epsilon \Sigma_{2}$. In view of this, if $\left\{S_{i}\right\}_{i=1}^{n}$ is a partition of $\Sigma_{2}$, one has

$$
\begin{aligned}
\sum_{i=1}^{n}\left\|F_{2}\left(S_{1}\right)\right\| & \leqslant \sum_{i=1}^{n} \liminf _{\pi}\left\|F_{2_{\tau(x)}}\left(S_{i}^{Y}\right)\right\| \\
& \leqslant \liminf _{\pi} \sum_{i=1}^{n}\left\|F_{2_{\tau(\pi)}}\left(S_{i}\right)\right\| \leqslant \underset{\pi}{\liminf } \mathscr{V}\left(F_{z_{\tau(\pi)}}\right) .
\end{aligned}
$$

But

$$
\mathscr{V}\left(F_{2_{\tau(\pi)}}\right)=\sum_{\pi}\left\|F_{2}\left(\tau E_{i}\right)\right\|=\sum_{\pi}\left\|F^{\prime}\left(E_{i}\right)\right\| \leqslant \mathscr{V}\left(F^{\prime}\right)
$$

for $\pi=\left\{E_{i}\right\}$. Hence

$$
\underset{\pi}{\liminf } \mathscr{V}\left(F_{2_{\tau(x)}}\right) \leqslant \mathscr{V}\left(F^{\prime}\right)
$$

Since the partition $\left\{S_{i}\right\}$ is arbitrary, it follows, from (B) and the preceding inequality, that $\mathscr{V}\left(F_{2}\right) \leqslant \mathscr{V}(F)$. This and (A) imply that $\mathscr{V}\left(F_{2}\right)$ $=\mathscr{V}(F)$.

Finally, using the second part of the hypothesis, it will be shown that

$$
\left\{\frac{F_{2}(E)}{\mu_{2}(E)}: \frac{\left\|F_{2}(E)\right\|}{\mu_{2}(E)} \leqslant n\right\}=A_{n}
$$

is weakly sequentially compact for each positive integer $n$. In order to prove this, it is sufficient to show that $A_{n}$ is contained in the strong closure of

$$
\left\{\frac{F(E)}{\mu(E)}: \frac{\|F(E)\|}{\mu(E)} \leqslant n+1\right\}=B_{n+1}
$$

for each positive integer $n$. Suppose $E \epsilon \Sigma_{2}$ is such that $F_{2}(E) / \mu_{2}(E) \epsilon A_{n}$. If $E \in \Sigma_{1}$, then

$$
\frac{F_{2}(E)}{\mu_{2}(E)}=\frac{F_{2}\left(\tau\left(E_{0}\right)\right)}{\mu_{2}\left(\tau\left(E_{0}\right)\right)}
$$

for some $E_{0} \epsilon \Sigma,=F\left(E_{0}\right) / \mu\left(E_{0}\right) \epsilon B_{n_{+1}}$.

If $E \notin \Sigma_{1}$, the definition of $F_{2}$ establishes the existence of a sequence $\left\{E_{m}\right\} \subset \Sigma_{1}$ such that

$$
\lim _{m} F_{2}\left(E_{m}\right)=F_{2}(E) \text { (strongly) and } \quad \lim _{m} \mu_{2}\left(E_{m}\right)=\mu_{2}(E) .
$$

Since $\left\|F_{2}(E) / \mu_{2}(E)\right\| \leqslant n$, it follows that there exists $m_{0}$ such that

$$
\left\|\frac{F_{2}\left(E_{m}\right)}{\mu_{2}\left(E_{m}\right)}\right\| \leqslant n+1 \quad \text { for } \quad m \geqslant m_{0} .
$$

The above argument shows that $F_{2}\left(E_{m}\right) / \mu_{2}\left(E_{m}\right) \in B_{n+1}$. Moreover

$$
\lim _{m}\left\|\frac{F_{2}\left(E_{m}\right)}{\mu_{2}\left(E_{m}\right)}-\frac{F_{2}(E)}{\mu_{2}(E)}\right\|=0 .
$$

Hence $F_{2}(E) / \mu_{2}(E)$ belongs to the strong closure of $B_{n+1}$. It follows that $A_{n}$ is contained in the strong closure of $B_{n+1}$. Since $B_{n+1}$ is weakly sequentially compact by hypothesis, the Eberlein-Smulian theorem ([9], V. 6.1) implies $A_{n}$ is weakly sequentially compact. 
* In view of the established properties of $\mathrm{F}_{2}, \mathrm{~F}_{2}$ satisfies the hypothesis of Phillips' generalization of the classical Radon-Nikodym theorem to vector-valued functions $([16]$, p. 134). This theorem guarantees the existence of a unique $F \in L^{1}\left(S_{1}, \Sigma_{2}, \mu_{2}, \mathscr{X}\right)$ such that

$$
F_{2}\left(E_{2}\right)=\int_{\mathbb{E}_{2}} f d \mu_{2}
$$

for all $E_{2} \in \Sigma_{2}$. By virtue of the fact that $\Sigma_{2}$ is the $\sigma$-field generated by $\Sigma_{1}$, the $\Sigma_{1}$-simple functions are dense in $L^{1}\left(S_{1}, \Sigma_{2}, \mu_{2}, \mathscr{X}\right)$ ([9], III. 8.3). Therefore, if $\varepsilon>0$ is given, there exists a $\Sigma_{1}$-simple function

$$
f_{\varepsilon}=\sum_{i=1}^{n} \alpha_{i} \chi_{I_{i}}, \quad \alpha_{i} \in X, E_{i} \in \Sigma_{1}, H_{i} \cap E_{j}=\varnothing, i \neq i,
$$

satisfying

$$
\int_{S_{1}}\left\|f-f_{8}\right\| d \mu_{2}<\varepsilon
$$

If $F_{\varepsilon}$ is the indefinite integral of $f_{\varepsilon}$, then $\mathscr{V}^{\prime}\left(F_{2}-W_{\varepsilon}\right)<\varepsilon$ by [9], III. 2.20. Hence

$$
\mathscr{V}\left(\left(F_{2}-F_{s}\right) \mid \Sigma_{1}\right)<\mathscr{V}\left(H_{2}-H_{8}\right)<\varepsilon
$$

where $\left(F_{2}-F_{s}\right) \mid \Sigma_{1}$ is the restriction of $F_{2}-F_{s}$ to $\Sigma_{1}$. But $F_{2}\left(W_{1}\right)$ $=F\left(\tau^{-1}\left(E_{1}\right)\right)$ for all $E_{1} \epsilon \Sigma_{1}$. Therefore if $H_{8}$ is the indefinite integral of

$$
h_{\varepsilon}=\sum_{i=1}^{n} \alpha_{i} \chi \tau^{-1}\left(E_{i}\right)
$$

then $H_{\theta}(E)=F_{\theta}(\tau(E))$ for all $E \in \Sigma$. Hence

$$
\mathscr{N}\left(F-\int_{(\cdot)} h_{s} d \mu\right)=\mathscr{V}\left(\left(F_{2}-F_{6}\right) \mid \Sigma_{1}\right)<\varepsilon, \quad \text { q. e. d. }
$$

The hypothesis of theorem 3 cannot be materially weakened. If the condition on the set function were deleted, the resulting statement would be false. Indeed, if $\Sigma$ were a $\sigma$-field and $\mu$ were countably additive and finite on $\Sigma$, the truth of this statement would imply that each $\mathscr{X}$-valued $\mu$-continuous countably additive set function had a representation as an indefinite Bochner integral. A counter example cited before also applies to this situation and can be found in [23]; p. 135. However, if the conditions on $F$ are relaxed, but the conditions on $\mathscr{X}$ are strengthened, then the following result may be stated.

The following corollary is basic to the main result of the second part of this section.
CoROLlary 4. Let $\mathscr{X}$ be a reflexive $B$-space. Let $\mu$ be a non-negative element of $\mathrm{ba}(\Omega, \Sigma, C)$ and $F \epsilon \mathrm{ba}(\Omega, \Sigma, \mathscr{X})$ be $\mu$-continuous. Then for each $\varepsilon>0$, there is a $\mu$-simple function $f_{\varepsilon}$ such that

$$
\mathscr{V}\left(F(\cdot)-\int_{(\cdot)} f_{\varepsilon} d \mu\right)<\varepsilon .
$$

Consequently, if $\mu(\Omega)<\infty$ and $\mathscr{X}$ is reflexive, step functions are dense in $\nabla^{1}(\Omega, \Sigma, \mu, \mathscr{X})$.

Proof. The first assertion immediately follows from the fact that boundedness and weak sequential compactness are equivalent in a reflexive $B$-space. The second assertion follows from the facts that step functions in $V^{1}(\mathscr{X})$ are merely indefinite integrals of $\mu$-simple functions and that the norm of $V^{1}(\mathscr{X})$ is the variation norm $\mathscr{V}(\cdot)$, q. e. d.

\section{The structure of $V^{\Phi}(\mathscr{X})$}

The principal results of this section deal with the closed subspace of $V^{\Phi}(\mathscr{X})$ determined by step functions. The main result here shows that step functions are dense in $V^{\Phi}(\mathscr{X})$ if $\Phi$ obeys the $\Delta_{2}$-condition and $\mathscr{X}$ is a reflexive Banach space.

Throughout this section $\mathscr{X}$ is a Banach space, $\Phi$ a continuous Young's function, $\Omega$ a point set, $\Sigma$ a field of subsets of $\Omega, \mu$ an finitely additive extended real-valued non-negative set function defined for $E \epsilon \Sigma \cdot \Sigma_{0} \subset \Sigma$ is the ring of sets of finite $\mu$-measure.

Definition 1. $S^{\Phi}(\Omega, \Sigma, \mu, \mathscr{X})\left(=S^{\Phi}(\mathscr{X})\right)$ is the closed subspace of $\nabla^{\Phi}(\Omega, \Sigma, \mu, \mathscr{X})\left(=\nabla^{\Phi}(\mathscr{X})\right)$ determined by step functions. (If $\Phi(x)$ $=|x|, S^{\Phi}(\mathscr{X})$ will be denoted by $\left.S^{1}(\mathscr{X})\right)$.

THEOREM 2. If $F$ belongs to $S^{\Phi}(\mathscr{X})$, then

$$
\lim _{\pi} N_{\Phi}\left(F-F_{\pi}\right)=0 .
$$

Proof. Clearly $\lim F_{\pi}=F$ in $V^{\Phi}(\mathscr{X})$ for all step functions $F$. Moreover, $N_{\Phi}\left(F_{\pi}\right) \leqslant N_{\Phi}\left(F^{\pi}\right)^{\pi}$ for all $F \epsilon S^{\Phi}(\mathscr{X})$ and all partitions $\pi$. An application of [9], II. 1.18, yields the result, q. e. d.

Lemma 3. (a) If $F$ belongs to $S^{\mathbf{1}}(\mathscr{X})$, then $\mathscr{V}(F, \cdot)$, the variation of $F$, belongs to $V^{1}(R)$ where $R$ is the real line.

(b) If $F$ belongs to $V^{\Phi}(\mathscr{X})$, then $I_{\Phi}(\mathscr{V}(F, \cdot))=I_{\Phi}(F)$.

Proof. (a) $\mathscr{V}(F, \cdot)$ is clearly additive and of bounded variation. It remains to show that $\mathscr{V}(F, \cdot)$ is $\mu$-continuous. Since $F \in S^{1}(\mathscr{X})$, for each $\varepsilon>0$, there exists a partition $\pi_{0}$ such that

$$
\mathscr{V}\left(F-F_{n}\right)=N_{1}\left(F-F_{\pi}\right)<\varepsilon \text { for } \pi \geqslant \pi_{0} .
$$


Thus for any partition $\Delta=\left\{E_{n}\right\}$

$$
\begin{aligned}
\sum_{\Delta}\left|\mathscr{V}\left(F, E_{n}\right)-\mathscr{V}\left(F_{n}, E_{n}\right)\right| & \leqslant \sum_{\Delta} \mathscr{V}\left(F \cdot E_{n}-F_{n} \cdot W_{n}\right) \\
=\sum_{\Delta} \mathscr{V}\left(\left(F-F_{n}\right) \cdot E_{n}\right) & \leqslant \mathscr{V}\left(F-F_{n}^{\prime}\right)=N_{1}\left(F^{\prime}-F_{n}^{\prime}\right) \quad \text { for } \quad \pi \geqslant \pi_{0} .
\end{aligned}
$$

Hence $\lim \mathscr{V}\left(F_{n}, \cdot\right)=\mathscr{V}\left(F^{\prime}, \cdot\right)$ strongly in $A^{1}(R)$. But each. $\mathscr{V}\left(F_{n}^{\prime}, \cdot\right)$ is evidently ${ }^{\pi} \mu$-continuous and consequently is in $V^{1}(R)$. The conclusion follows directly from theorem I. 16.

(b) Let $F \in V^{\Phi}(\mathscr{C})$. From the definition of $\mathscr{V}\left(F^{\prime}, \cdot\right)$, it is seen that $\lim \mathscr{V}\left(F_{x}, E\right)=\mathscr{V}(F, E)$ for each $E \in \Sigma_{0}$. In addition,

$$
\mathscr{V}\left(F_{\pi}, \cdot\right)=\sum_{\pi} \frac{\left\|F^{\prime}\left(E_{n}\right)\right\|}{\mu\left(E_{n}\right)} \mu \cdot E_{n} ;
$$

from this and theorem I. 9, it follows that

$$
I_{\Phi}\left(\mathscr{V}\left(F_{n}, \cdot\right)\right)=I_{\Phi}\left(F_{n}\right) \leqslant I_{\Phi}\left(F^{\prime}\right) .
$$

These facts combined with the lower semi-continuity of $I_{\Phi}(\cdot)$ (theorem I. 7) imply

$$
I_{\Phi}(\mathscr{V}(F, \cdot)) \leqslant \liminf _{\pi} I_{\Phi}\left(\mathscr{V}\left(F_{\pi}, \cdot\right)\right) \leqslant I_{\Phi}\left(I^{\prime}\right)
$$

To prove the reverse inequality, note that $\mathscr{V}\left(F^{\prime}, E\right) \geqslant\|F(E)\|$ for each $E \in \Sigma_{0}$. The monotonicity of $\Phi$ yields the inequality $I_{\Phi}\left(\mathscr{V}\left(F^{\prime}, \cdot\right)\right)$ $\geqslant I_{\Phi}(F)$ and the result, q. e. d.

Lemma 4. If $\mathbb{F} \in V^{\Phi}(\mathscr{X})$ and $F^{1} \in \in \in S^{1}(\mathscr{X})$ for each $E \in \Sigma_{0}$, then $I_{\Phi}\left(\mathscr{V}\left(F^{\prime}, \cdot\right), \cdot\right) \in V^{1}(R)$ (where $I_{\Phi}(\mathscr{V}(F, \cdot), \cdot)$ has the value $I_{\Phi}\left(\mathscr{V}\left(F^{\prime}, \cdot\right), E\right)$ for each $\left.E \in \Sigma_{0}\right)$. Consequently, $I_{\Phi}(F, \cdot) \in V^{1}(R)$.

Proof. For notational convenience the set function $\mathscr{N}\left(F^{\prime}, \cdot\right)$ will be denoted by $G$. From lemma $3(\mathrm{~b})$ it follows that $I_{\phi}(G, W)$ is finite for each $E \epsilon \Sigma_{0}$. That $I_{\phi}(G, \cdot)$ is additive on $\Sigma_{0}$ is assured by lemma T. 6 . Clearly $I_{\Phi}(G, \cdot)$ is of bounded variation and vanishes on $\eta$-null sets. Hence $I_{\Phi}(G, \cdot) \in A^{1}(R)$. To show that $I_{\Phi}(G, \cdot) \in V^{1}(R)$, it must be shown that $I_{\Phi}(G, \cdot)$ is $\mu$-continuous. By hypothesis, $H^{\cdot} \cdot W_{i} \in S^{1}(\mathscr{X})$ for all $Z \in \Sigma_{0}$. From lemma $3\left(\right.$ a),$G \cdot E \in V^{1}(R)$ for all $E \in \Sigma_{0}$. Hence by a theorem of J3ochner and Phillips [6],

$$
\lim _{n \rightarrow \infty}(G \wedge n \mu) \cdot E=\lim _{n} G \cdot E \wedge n \mu=G \cdot E
$$

strongly in $V^{1}(R)$ for $E \epsilon \Sigma_{0}$ where $G \wedge n \mu$ is the set function defined by

$$
G \wedge n \mu(E)=\inf _{A_{\subset} E}\{G(A)+\mu(E-A): A \epsilon \Sigma\} .
$$

The lower semicontinuity of $I_{\Phi}(\cdot)$ implies

$$
I_{\Phi}(G, E) \leqslant \liminf _{n} I_{\Phi}(G \wedge n \Phi, E) ;
$$

on the other hand, the monotonicity of $\Phi$ insures $I_{\Phi}(G, E) \geqslant I_{\Phi}(G \wedge n \mu, E)$ for each $E \epsilon \Sigma_{0}$. Hence

$$
I_{\Phi}(G)=\lim _{n} I_{\Phi}(G \wedge n \mu)
$$

and if $\pi=\left\{E_{n}\right\}$ is any partition of $\Omega$ and $A=\bigcup_{n} E_{n}$, then

$$
\begin{aligned}
\sum_{\pi} \mid I_{\Phi}(G, & \left.E_{n}\right)-I_{\Phi}\left(G \wedge n \mu, E_{n}\right) \mid \\
& =\sum_{\pi}\left(I_{\Phi}\left(G, E_{n}\right)-I_{\Phi}\left(G \wedge n \mu, E_{n}\right)\right) \\
& =\sum_{\pi} I_{\Phi}\left(G, E_{n}\right)-\sum_{\pi} I_{\Phi}\left(G \wedge n \mu, E_{n}\right) \\
& \leqslant I_{\Phi}(G, A)-I_{\Phi}(G \wedge n \mu, A)+I_{\Phi}\left(G, A^{\prime}\right)-I_{\Phi}\left(G \wedge n \mu, A^{\prime}\right) \\
& =I_{\Phi}(G)-I_{\Phi}(G \wedge n \mu) .
\end{aligned}
$$

Since the partition $\pi$ is arbitrary,

and

$$
N_{1}\left(I_{\Phi}(G, \cdot)-I_{\Phi}(G \wedge n \mu, \cdot)\right) \leqslant I_{\Phi}(G)-I_{\Phi}(G \wedge n \mu),
$$

$$
\lim _{n} I_{\Phi}(G \wedge n \mu, \cdot)=I_{\Phi}(G, \cdot)
$$

strongly in $A^{1}(R)$. But $I_{\Phi}(G \wedge n \mu, E) \leqslant \Phi(n) \mu(E)$ for each $E \epsilon \Sigma_{0}$. Hence $I_{\Phi}(G \wedge n \mu, \cdot)$ is $\mu$-continuous. Therefore $I_{\Phi}(G, \cdot) \in V^{1}(R)$ because $V^{1}(R)$ is a closed subspace of $A^{1}(R)$. The last statement follows immediately from the above and lemma 3 (b), q. e. d.

LEMmA 5. (a) If $F \in V^{\Phi}(\mathscr{X})$, then for each choice of $\varepsilon>0$, there exists a set $A \in \Sigma_{0}$ such that $I_{\Phi}\left(F, A^{\prime}\right)<\varepsilon$.

(b) If $F \in V^{\Phi}(\mathscr{X})$ and $F \cdot E \in S^{1}(\mathscr{X})$ for each $E$ in $\Sigma_{0}$, then

$$
I_{\Phi}(F, \cdot)=\lim _{\pi} I_{\Phi}\left(F_{\pi}, \cdot\right)
$$

strongly in $V^{1}(R)$.

Proof. (a) Let $\varepsilon>0$ be given. According to lemma I. 5, there exists a partition $\pi=\left\{E_{n}\right\}$ such that

$$
0 \leqslant I_{\Phi}(F)-I_{\Phi}\left(F_{\pi}\right)<\varepsilon .
$$

Let $A=\bigcup_{n} E_{n}$. Then $A \in \Sigma_{0}$ and $I_{\Phi}\left(F_{n}\right) \leqslant I_{\Phi}(F, A) \leqslant I_{\Phi}(F)$. Whence

$$
I_{\Phi}\left(F, A^{\prime}\right)=I_{\Phi}(F)-I_{\Phi}(F, A) \leqslant I_{\Phi}(F)-I_{\Phi}\left(F_{\pi}\right)<\varepsilon .
$$


This proves (a).

(b) First note that if $E \epsilon \Sigma_{0}$ and $\mu(E) \neq 0$, thesi

hence

$$
\Phi\left(\frac{\|F(E)\|}{\mu(E)}\right) \mu(E) \leqslant I_{\Phi}(F, E)
$$

$$
\Phi\left(\frac{\|F(E)\|}{\mu(E)}\right) \leqslant \frac{I_{\Phi}(F, E)}{\mu(E)} .
$$

In view of this inequality, if $\pi=\left\{\mathbb{H}_{n}\right\}$ is any partition, one has

$$
I_{\Phi}\left(F_{\pi}, \cdot\right)=\sum_{\pi} \Phi\left(\frac{\left\|F\left(E_{n}\right)\right\|}{\mu\left(E_{n}\right)}\right) \mu \cdot E_{n} \leqslant \sum_{\pi} \frac{I_{\phi}\left(W^{\prime}, E_{n}\right)}{\mu\left(\mathbb{E}_{n}\right)} \mu \cdot H_{n}=I_{\Phi}(\not T, \cdot)_{\pi}
$$

so that

$$
I_{\Phi}\left(F_{n}, \cdot\right) \leqslant I_{\Phi}\left(F^{\prime}, \cdot\right)_{n}
$$

for all partitions $\pi$. Now for any partition $\pi$,

$$
\begin{aligned}
N_{1}\left(I_{\Phi}(F, \cdot)\right. & \left.-I_{\Phi}\left(F_{\pi}, \cdot\right)\right) \\
& \leqslant N_{1}\left(I_{\Phi}(F, \cdot)-I_{\Phi}(F, \cdot)_{\pi}\right)+N_{1}\left(I_{\Phi}\left(F^{\prime}, \cdot\right)_{\pi}-I_{\Phi}\left(F_{\pi}, \cdot\right)\right) \\
& =N_{1}\left(I_{\Phi}(F, \cdot)-I_{\Phi}(F, \cdot)_{\pi}\right)+I_{\Phi}(F, \cdot)_{\pi}(\Omega)-I_{\Phi}\left(H_{\pi}^{\prime}, \cdot\right)(\Omega),
\end{aligned}
$$

since $I_{\Phi}\left(F_{\pi}, E\right) \leqslant I_{\Phi}(F, E)_{\pi}$ for all $E \in \Sigma_{0}$ and the variation of a nonnegative set functicn is its value on the whole set $\Omega$. But

and

$$
I_{\Phi}(F, \cdot)_{\pi}(\Omega)=I_{\Phi}(F, \Omega)=I_{\Phi}\left(F^{\prime}\right),
$$

Hence

$$
I_{\Phi}\left(H_{\pi}, \cdot\right)(\Omega)=I_{\Phi}\left(H_{\pi}, \Omega\right)=I_{\Phi}\left(F_{\pi}\right)
$$

$$
\lim _{\pi}\left(I_{\Phi}\left(F^{\prime}\right)-I_{\Phi}\left(F_{\pi}\right)\right) \leqslant \lim _{\pi} N_{1}\left(I_{\Phi}(F, \cdot)-I_{\Phi}(F, \cdot)_{\pi}\right)+0
$$

by theorem I. 9. Now let $\varepsilon>0$ be given. By part (a), there exists on $A \in \Sigma_{0}$ such that $I_{\Phi}\left(F, A^{\prime}\right)<\varepsilon$. For any partition $\pi$ refining the trivial partition $\{A\}$, one has

$$
\begin{aligned}
N_{1}\left(I_{\Phi}\left(F^{\prime}, \cdot\right)-I_{\Phi}\left(F^{\prime}, \cdot\right)_{\pi}\right) \\
\quad \leqslant N_{1}\left(I_{\Phi}(F, \cdot) \cdot A-I_{\Phi}(F, \cdot)_{\pi} \cdot A\right)+N_{1}\left(I_{\Phi}(F, \cdot) \cdot A^{\prime}\right)+N_{1}\left(I_{\Phi}\left(F^{\prime}, \cdot\right)_{\pi} \cdot A\right) \\
\quad \leqslant N_{1}\left(I_{\Phi}(F, \cdot) \cdot A-I_{\Phi}(F, \cdot)_{\pi} \cdot A\right)+2 N_{1}\left(I_{\Phi}\left(F^{\prime}, \cdot\right) \cdot A^{\prime}\right) \\
\quad=N_{1}\left(I_{\Phi}\left(F^{\prime}, \cdot\right) \cdot A-I_{\Phi}\left(F^{\prime}, \cdot\right)_{\pi} \cdot A\right)+2 I_{\Phi}\left(F^{\prime}, A^{\prime}\right) \\
\quad \leqslant N_{1}\left(I_{\Phi}\left(F^{\prime}, \cdot\right) \cdot A-I_{\Phi}(F, \cdot)_{\pi} \cdot A\right)+\varepsilon
\end{aligned}
$$

by the choice of $A$.
Now $I_{\Phi}(F, \cdot) \cdot A \in V^{1}(R)$ by lemma 4. Since $\mu(A)<\infty$, corollary III. 5 implies

$$
\lim _{\pi} N_{1}\left(I_{\Phi}(F, \cdot) \cdot A-I_{\Phi}(F, \cdot)_{\pi} \cdot A\right)=0
$$

Since $\varepsilon>0$ is arbitrary, this implies

$$
\lim _{\pi} N_{1}\left(I_{\Phi}(F, \cdot)-I_{\Phi}(F, \cdot)_{\pi}\right)=0,
$$

which, in view of the above inequalities, implies

$$
\lim _{\pi} N_{1}\left(I_{\Phi}(F, \cdot)-I_{\Phi}\left(F_{n}, \cdot\right)\right)=0, \quad \text { q. e. d. }
$$

The principal results of this section are the following theorem and its corollaries.

THEOREM 6. If $F \in V^{\Phi}(\mathscr{X})$ satisfies (i) $I_{\Phi}\left(a F^{\prime}\right)<\infty$ for all scalars $a$, and (ii) $H^{1}: E \in S^{1}(\mathscr{X})$ for all $E \epsilon \Sigma_{0}$, then

$$
\lim _{\pi} N_{\Phi}\left(F-F_{\pi}\right)=0
$$

Proof. First it will be shown $\lim I_{\Phi}\left(F-F_{n}\right)=0$. Let $\varepsilon>0$ be given. According to lemma $5\left(\right.$ a), there exists a set $A \in \Sigma_{0}$ such that $I_{\Phi}\left(2 F, A^{\prime}\right)<\varepsilon$. Now if $\{A\} \leqslant \pi_{1} \leqslant \pi_{2}$, then

$$
I_{\Phi}\left(F_{\pi_{2}}-F_{\pi_{1}}, A^{\prime}\right)=I_{\Phi}\left(\mathscr{V}\left(F_{n_{2}}-F_{\pi_{1}} \cdot\right), A^{\prime}\right)
$$

by lemma 4 ,

$$
\begin{aligned}
& \leqslant I_{\Phi}\left(\mathscr{V}\left(F_{\pi_{2}}, \cdot\right)+\mathscr{V}\left(F_{\pi_{1}}^{\prime} \cdot \cdot\right), A^{\prime}\right) \\
& \leqslant I_{\Phi}\left(2 \mathscr{V}(F, \cdot), A^{\prime}\right) \text { by monotonicity of } \Phi, \\
& =I_{\Phi}\left(2 F^{\prime}, A^{\prime}\right), \text { by lemma } 4, \\
& <\varepsilon \text { by the choice of } A .
\end{aligned}
$$

Hence

$$
I_{\Phi}\left(F-F_{\pi_{1}}, A^{\prime}\right) \leqslant \liminf _{\pi_{2}} I_{\Phi}\left(F_{\pi_{2}}-F_{\pi_{1}}, A^{\prime}\right)<\varepsilon
$$

Therefore, it can and will be assumed that $\mu(\Omega)<\infty$.

Let $\delta>0$ be given and fix $\gamma>0$ such that $\Phi(\gamma) \mu(\Omega)<\delta / 2$. For each partition $\pi$, form the $\mu$-simple function

Consider

$$
f_{n}=\sum_{n} \frac{F\left(E_{n}\right)}{\mu\left(E_{n}\right)} \chi_{E_{n}}
$$

$$
\int_{\Omega}\left\|f_{\pi_{1}}-f_{\pi_{2}}\right\| d \mu=N_{1}\left(F_{\pi_{1}}-F_{\pi_{2}}\right)
$$


The facts $W^{1} \cdot E \epsilon S^{1}(\mathscr{X})$ for every $E \epsilon \Sigma_{0}$ and $\mu(\Omega)<\infty$ imply $W^{\prime} \epsilon S^{1}(\mathscr{X})$. Consequently, from theorem 2, it follows that

$$
0=\lim _{\pi_{1}, \pi_{2}} N_{1}\left(F_{\pi_{1}}-F_{\pi_{2}}\right)=\lim _{\pi_{1}, \pi_{2}} \int_{\Omega}\left\|f_{\pi_{1}}-f_{\pi_{2}}\right\| d \mu .
$$

If $E_{\pi_{1}, \pi_{2}}=\left\{\omega:\left\|f_{\pi_{1}}(\omega)-f_{\pi_{2}}(\omega)\right\| \geqslant \gamma\right\}$, then $E_{\pi_{1}, \pi_{2}} \in \Sigma$ and

$0=\lim _{\pi_{1}, \pi_{2}} \int_{\Omega}\left\|f_{\pi_{1}}-f_{\pi_{2}}\right\| d \mu \geqslant \lim _{\pi_{1}, \pi_{2}} \int_{x_{\pi_{1}, \pi_{2}}}\left\|f_{\pi_{1}}-f_{\pi_{2}}\right\| d \mu \geqslant \lim _{\pi_{1}, \pi_{2}} \gamma \mu\left(E_{\pi_{1}, \pi_{2}}\right) \geqslant 0$.

Whence $\lim \mu\left(E_{\pi_{1}, \pi_{2}}\right)=0$.

Now consider for $\pi_{1} \leqslant \pi_{2}$

$$
\begin{aligned}
I_{\Phi}\left(F_{\pi_{2}}-F_{\pi_{1}}\right) & =I_{\Phi}\left(F_{\pi_{2}}-F_{\pi_{1}}, E_{\pi_{1}, \pi_{2}}^{\prime}\right)+I_{\Phi}\left(F_{\pi_{2}}-F_{\pi_{1}}, E_{\pi_{1}, \pi_{2}}\right) \\
& \leqslant \Phi(\gamma) \mu\left(E_{\pi_{1}, \pi_{2}}^{\prime}\right)+I_{\Phi}\left(F_{\pi_{2}}-F_{\pi_{2}}, E_{\pi_{1}, \pi_{2}}\right) \\
& \leqslant \Phi(\gamma) \mu(\Omega)+I_{\Phi}\left(\mathscr{V}\left(F_{\pi_{2}}-F_{\pi_{1}}\right), E_{\pi_{1}, \pi_{2}}\right)
\end{aligned}
$$

by lemma $3(\mathrm{~b})$,

$$
\begin{aligned}
& \leqslant \delta / 2+I_{\Phi}\left(\mathscr{V}\left(F_{\pi_{2}}, \cdot\right)+\mathscr{V}\left(F_{\pi_{1}}, \cdot\right), E_{\pi_{1}, \pi_{2}}\right) \\
& \leqslant \delta / 2+I_{\Phi}\left(2 \mathscr{V}(F), E_{\pi_{1}, \pi_{2}}\right), \quad \text { by monotonicity of } \Phi, \\
& =\delta / 2+I_{\Phi}\left(2 F, E_{\pi_{1}, \pi_{2}}\right), \quad \text { lemma } 3(\mathrm{~b}) .
\end{aligned}
$$

But $\lim _{\pi_{1}, \pi_{2}} \mu\left(E_{\pi_{1}, \pi_{2}}\right)=0$. By the $\mu$-continuity of $I_{\Phi}(2 F, \cdot)$ (lemma 4) there exists a partition $\pi_{0}$ such that

Whence

$$
I_{\Phi}\left(2 F, E_{\pi_{1}, \pi_{2}}\right)<\delta / 2 \quad \text { for } \quad \pi_{2} \geqslant \pi_{1} \geqslant \pi_{0} .
$$

$$
I_{\Phi}\left(F_{\pi_{2}}-F_{\pi_{1}}\right)<\delta / 2+\delta / 2=\delta
$$

for $\pi_{2} \geqslant \pi_{1} \geqslant \pi_{0}$. This combined with the lower semi-continuity (theorem I. 7) of $I_{\Phi}(\cdot)$ yields

$$
I_{\Phi}\left(F-F_{\pi_{1}}\right) \leqslant \liminf _{\pi_{2}} I_{\Phi}\left(F_{\pi_{2}}-F_{\pi_{1}}\right) \leqslant \delta \quad \text { for } \quad \pi_{1} \geqslant \pi_{0} .
$$

Since $\delta>0$ is arbitrary, it follows that $\lim I_{\infty}\left(F-F_{\pi}\right)=0$. To com. plete the proof of the theorem, note that if ${ }^{\pi} F$ satisfies the hypothesis of the theorem, so does $F / k$ for any $k>0$. From the above analysis for each $k>0$, there exists $\pi_{k}$ such that

$$
I_{\Phi}\left(\frac{F-F_{\pi}}{k}\right)=I_{\Phi}\left(\frac{F}{k}-\frac{F_{\pi}}{k}\right) \leqslant 1
$$

for all $\pi \geqslant \pi_{k}$. From the definition of $N_{\Phi}(\cdot)$, one has

$$
N_{\Phi}\left(F-F_{n}\right) \leqslant k
$$

for $\pi \geqslant \pi_{k}$. Since $k>0$ is arbitrary, it follows that

$$
\lim _{\pi} N_{\Phi}\left(F-F_{\pi}\right)=0, \quad \text { q. e. } d \text {. }
$$

Combining the results of section 1 with those of this section given the following result which will play a major role in the presentation theorems of section $\mathrm{V}$.

CoRollary 7. If $\Phi$ obeys the $\Delta_{2}$-condition and $\mathscr{X}$ is reflexive, then $S^{\Phi}(\mathscr{X})=\nabla^{\Phi}(\mathscr{X})$.

Proof. Let $F \in V^{\Phi}(\mathscr{X})$. From lemma I. 13 and corollary III. 4 it follows that $F \cdot E \in S^{1}(\mathscr{X})$ for all $E \in \Sigma_{0}$. Since $\Phi$ obeys the $\Delta_{2}$-condition $I_{\Phi}\left(a F^{\prime}\right)<\infty$ for all scalars $a$. Hence $F \in S^{\Phi}(\mathscr{X})$, q. e. d.

Applying the preceding results to the $L^{\Phi}(\mathscr{X})$-spaces, one has the following theorem:

THEOREM 8. If $\Phi$ obeys the $\Delta_{2}$-condition, then $\mu$-simple functions are dense in $L^{\Phi}(\mathscr{X})$.

Proof. Let $f \epsilon L^{\Phi}(\mathscr{X})$ and $\lambda$ be the isometric injection of $L^{\Phi}(\mathscr{X})$ into $V^{\Phi}(\mathscr{X})$ of theorem II. 5. Since $\mu$-simple functions are dense in $L^{\mathbf{1}}(\mathscr{X})$ by [9], III. 3.8, it follows that $\lambda f \cdot E \in S^{1}(\mathscr{X})$ for each $E \in \Sigma_{0}$. The $\Delta_{2}$-condition ensures that $I_{\Phi}(a \lambda f)$ is finite for all scalars $a$. Hence, by theorem 6 ,

$$
\lim _{\pi} N_{\Phi}\left(\lambda f-(\lambda f)_{\pi}\right)=0 \text {. }
$$

Since $\lambda^{-1}(\lambda f)_{\pi}$ is a $\mu$-simple function and $\lambda$ is an isometry, the conclusion immediately follows, q. e. d.

Remark. If $\Phi(x)=|x|^{p}, 1 \leqslant p<\infty$, this result was proved in [9], III. 3.8. Generalizing that method, one can also obtain an independent proof for this theorem.

\section{v. Linear operators on $\nabla^{\Phi}(\mathscr{X})$}

In this section, the general bounded linear operator from $S^{\Phi}(\mathscr{X})$ to an arbitrary $B$-space $\mathscr{Y}$ is characterized in terms of a certain "integral" representation involving operator-valued set functions. Then it is shown that the space of such operators is isometrically isomorphic to a space of operator-valued set functions $W^{\Psi}(B(\mathscr{X}, \mathscr{Y}))$ which is related to a $V^{\Psi}\left(\mathscr{X}^{*}\right)$-space in a natural way. As corollaries specializations of these results are given for operators on $L^{\Phi}(\mathscr{X})$.

The problem of representing bounded linear operators on $L^{p}(\mathscr{X})$, $1 \leqslant p<\infty$, to an arbitrary $B$-space has been a recurring one. Although some special cases have been resolved $[4,7,16,17]$, only partial results have been obtained in the case of the general bounded linear operator on $L^{\Phi}(\mathscr{X})$. One of the papers cited above, the paper of Bochner and Taylor 
[7] allows itself to be abstracted to the $\nabla^{\Phi}(\mathscr{X})$ setting. It is this paper and [13] which motivate the considerations of this section.

Let $\Omega, \Sigma, \Sigma_{0}$, and $\mu$ be as in section I. $\mathscr{X}$ and $\mathscr{O}$ are $B$-spaces with conjugate spaces $\mathscr{X}^{*}$ and $\mathscr{Y}^{*}$ respectively. $B(\mathscr{X}, \mathscr{Y})$ is the space of bounded linear operators from $\mathscr{X}$ into $\mathscr{Y}$ with uniform norm. $\Phi$ is a continuous Young's function. The first lemma is concerned with associating an operator-valued set function with a bounded linear operator on $S^{\Phi}(\mathscr{X})$.

Lenara 1. Let $h \in B\left(S^{\Phi}(\mathscr{X}), \mathscr{Y}\right)$. Then there exists a unique finitely additive $B(\mathscr{X}, \mathscr{Y})$-valued $\mu$-continuous set function $H$, defined on $\Sigma_{0}$ and satisfying

$$
h\left(F^{\prime}\right)=\lim _{\pi} \sum_{\pi} \frac{H\left(\mathbb{H}_{n}\right)\left[H^{\prime}\left(\mathbb{E}_{n}\right)\right]}{\mu\left(\mathbb{E}_{n}\right)}
$$

for all $F \in S^{\Phi}(\mathscr{X})$, where the limit is taken in the Moore-Smith sense through all partitions $\pi$ of $\Omega$.

Proof. If $x_{1}, x_{2} \in \mathscr{X}$ are arbitrary and $a, b$ are any scalars, then $h\left(\left(a x_{1}+b x_{2}\right) \mu \cdot E\right)=h\left(a x_{1} \mu \cdot E\right)+h\left(b x_{2} \mu \cdot E\right)=a h\left(x_{1} \mu \cdot E\right)+b h\left(x_{2} \mu \cdot E\right)$ for any $E \in \Sigma_{0}$. Hence the set function $H$ defined for each $E$ in $\Sigma_{0}$ by

$$
H(\mathbb{E})=h(\cdot \mu \cdot E)
$$

is a linear operator from $\mathscr{X}$ to $\mathscr{Y}$ satisfying $H(E)[x]=h(x \mu \cdot E)$ for all $x \in \mathscr{X}$ and each $E \in \Sigma_{0}$.

Now consider

$$
\begin{aligned}
\|h(E)[x]\| & =\| h\left(x \mu \cdot E\|\leqslant\| h \| N_{\Phi}(x \mu \cdot E)\right. \\
& =\|h\|\|x\| N_{\Phi}(\mu \cdot E)=\|h\|\|x\| \inf \left\{K>0: I_{\Phi}\left(\frac{\mu \cdot E}{K}\right) \leqslant 1\right\} \\
& =\|h\|\|x\| \inf \left\{K>0: \Phi\left(\frac{1}{K}\right) \mu(E) \leqslant 1\right\}
\end{aligned}
$$

for any $x \in \mathscr{X}, E \in \Sigma_{0}$. This shows first that

$$
H(E) \epsilon B(\mathscr{X}, \mathscr{Y}) \text { for each } E \in \Sigma_{0},
$$

and second that

$$
\begin{gathered}
\lim _{\mu(\mathbb{E}) \rightarrow 0}\|H(\mathbb{E})\|_{\mathcal{B ( X}, \mathscr{Q})} \\
\leqslant\|h\| \lim _{\mu(\mathbb{E}) \rightarrow 0} \inf \left\{K>0: \Phi\left(\frac{1}{K}\right) \mu(E) \leqslant 1\right\}=0,
\end{gathered}
$$

since $\Phi(x)<\infty$ for $x<\infty$. To show $H$ is finitely additive, let $E_{1}, E_{2} \in \Sigma_{0}$, $E_{1} \cap E_{2}=\varnothing$. Then

$$
\begin{aligned}
H\left(E_{1} \cup E_{2}\right)[x] & =h\left(x \mu \cdot\left(E_{1} \cup E_{2}\right)\right) \\
& =h\left(x\left(\mu: E_{1}+\mu \cdot E_{2}\right)\right)=h\left(x \mu \cdot E_{1}\right)+h\left(x \mu \cdot E_{2}\right) \\
& =H\left(E_{1}\right)[x]+H\left(E_{2}\right)[x]=H\left(E_{1}\right)+H\left(E_{2}\right)[x],
\end{aligned}
$$

for all $x \in \mathscr{X}$. Therefore $H$ is finitely additive.

To establish representation (A), let $F \in S^{\Phi}(\mathscr{X})$. Then $\lim N_{\Phi}\left(F-F_{\pi}\right)$ $=0$ by theorem IV.2. The continuity of $h$ implies

$$
\lim _{\pi}\left\|h\left(F^{\prime}\right)-h\left(F_{\pi}\right)\right\| \leqslant\|h\| \lim _{\pi} N_{\Phi}\left(F-F_{\pi}\right)=0 .
$$

Thus $h(F)=\lim h\left(F_{n}\right)$. Now, the linearity of $h$ and the definition of $H$ yield

$$
\begin{aligned}
h\left(F_{\pi}\right) & =h\left(\sum_{\pi} \frac{F\left(E_{n}\right)}{\mu\left(E_{n}\right)} \mu \cdot E_{n}\right) \\
& =\sum_{\pi} \frac{h\left(F\left(E_{n}\right) \mu \cdot E_{n}\right)}{\mu\left(E_{n}\right)}=\sum_{\pi} \frac{H\left(E_{n}\right)\left[F\left(E_{n}\right)\right]}{\mu\left(E_{n}\right)}
\end{aligned}
$$

for any partition $\pi$ of $\Omega$. Therefore

$$
h(F)=\lim _{\pi} h\left(F_{\pi}\right)=\lim _{\pi} \sum_{\pi} \frac{H\left(E_{n}\right)\left[F\left(E_{n}\right)\right]}{\mu\left(E_{n}\right)} .
$$

The uniqueness is simple. For, if

$$
h\left(F^{\prime}\right)=\lim _{\pi} \sum_{\pi} \frac{H^{\prime}\left(E_{n}\right)\left[F^{\prime}\left(E_{n}\right)\right]}{\mu\left(E_{n}\right)}
$$

for all $F \in S^{\Phi}(\mathscr{X})$, then by the definition of $H$,

$$
H(E)[x]=h(x \mu \cdot E)=\lim _{n} \sum_{n} \frac{E^{\prime}\left(E_{n}\right)\left[x \mu\left(E_{n} \cap R E\right)\right]}{\mu\left(E_{n}\right)}=H^{\prime}(E)[x],
$$

for each $x \in \mathscr{X}$ and $E \in \Sigma_{0}$. Hence $H=H^{\prime}$, q. e. $d$.

Now that a representation of members of $B\left(S^{\Phi}(\mathscr{X}), \mathscr{Y}\right)$ in terms of operator-valued set functions has been established, one immediate question is this: what space of operator-valued set functions has a topology compatible with the uniform operator topology of $B\left(S^{\Phi}(\mathscr{C}), \mathscr{Y}\right)$ ? The following is devoted to defining a space of set functions $W^{\Psi}(B(\mathscr{X}, \mathscr{Y}))$ which is then shown to be isometrically isomorphic to $B\left(S^{\Phi}(\mathscr{X}), \mathscr{Y}\right)$. 
Definition 2. The space $W^{\Phi}(\Omega, \Sigma, \mu, B(\mathscr{X}, \mathscr{Y}))=W^{\mathscr{\Phi}}(B(\mathscr{X}, \mathscr{Y})$ consists of all finitely additive $\mu$-continuous $B(\mathscr{X}, \mathscr{O})$-valued set functions $H$ defined on $\Sigma_{0}$ which satisfy

(i) $y^{*} H \in V^{\Phi}\left(\mathscr{X}^{*}\right)$ for all $y^{*} \in X^{*}$.

(ii) $\sup _{\left\|\Psi^{*}\right\| \leq 1} N_{\Phi}\left(y^{*} H\right)<\infty$ where $y^{*} H$ is the set function defined for $E \in \Sigma_{0}$, by

$$
y^{*} H(E)[x]=y^{*}(H(E)[x]) .
$$

(Here $\Phi$ is an arbitrary Young's function).

The additivity of $H(\cdot)$ and the fact that $H(E) \in B(\mathscr{C}, \mathscr{Y})$ for $H \in \Sigma_{0}$ imply $y^{*} H(\cdot)$ is a finitely additive $\mathscr{C}^{*}$-valued set function on $\Sigma_{0}$; so (i) and (ii) are meaningful.

THEorem 3. The functional $\|\cdot\|_{W^{\Phi}}$ defined for $H \in W^{\Phi(B(\mathscr{X}, \mathscr{Q}))}$ by $\|H\|_{W^{\Phi}}$ $=\sup \left\{N_{\Phi}\left(y^{*} H\right): y^{*} \in Y^{*},\left\|y^{*}\right\| \leqslant 1\right\}$ is a norm, and $W^{\Phi}(B(\mathscr{X}, \mathscr{Y}))$ is a normed linear space.

Proof. If $H=0$, evidently $\|H\|_{W^{\Phi}}=\mathbf{0}$. On the other hand, if $H \neq 0$, then there exists $E \in \Sigma_{0}$ and $x \in \mathscr{X}$ such that $H(E)[x] \neq 0$. By a consequence of the Hahn-Banach theorem, there exists $y_{0}^{*}$ in the unit ball of $Y^{*}$ such that $y_{0}^{*} H(E)[x] \neq 0$. Consequently $y_{0}^{*} H \neq 0$ and $N_{\mathscr{\sigma}}\left(y_{0}^{*} H\right)>0$. Therefore $\|H\|_{p^{\infty} \Phi}>0$.

The other two properties of a norm follow directly from the corresponding properties of $N_{\Phi}$, q. e.d.

A proof of the completeness of $W^{\Phi}(B(\mathscr{X}, \mathscr{Y}))$ can be given in a way which is only a slight modification of the completeness proof for $A^{\mathscr{D}}(\mathscr{C})$, but the completeness of the $W^{\Phi}(B(\mathscr{X}, \mathscr{Y}))$ spaces of interest here is a consequence of the main theorem of this section. The next two lommas are preliminary to this result.

LEMMa 4. If $F \in V^{\Phi}(\mathscr{X})$, then $\|F\|_{\Phi} \leqslant I_{\Phi}(F)+1$.

Proof. If $\Psi$ is complementary to $\Phi$, by definition,

$$
\begin{aligned}
\left\|F^{\prime}\right\|_{\Phi} & =\sup _{N_{\Psi}(G) \leqslant 1} \sup _{\pi} \sum_{\pi} \frac{\left\|F\left(E_{n}\right)\right\|\left\|G\left(E_{n}\right)\right\|}{\mu\left(E_{n}\right)} \\
& \leqslant \sup _{N_{\Psi(}(G) \leqslant 1}\left(\sup _{\pi} \sum_{\pi} \Phi\left(\frac{\left\|F\left(E_{n}\right)\right\|}{\mu\left(I_{n}\right)}\right) \mu\left(E_{n}\right)+\sup _{\pi} \sum_{\pi} \Psi\left(\frac{\left\|G\left(E_{n}\right)\right\|}{\mu\left(E_{n}\right)}\right) \mu\left(I_{n}\right)\right) \\
& =\sup _{N_{\Psi(}(G) \leqslant 1}\left(I_{\Phi}(F)+I_{\Psi}(G)\right) \leqslant I_{\Phi}(F)+1, \quad \text { q. e. d. }
\end{aligned}
$$

LEMMA 5. If $x^{*} \in \mathscr{X}^{*}$ and $\varepsilon>0$ is arbitrary, there exists $x \in \mathscr{X}$ such that $x^{*}(x)=\left\|x^{*}\right\|$ and $|\|x\|-1|<\varepsilon$.

Pro of. This immediately follows from the definition of $\left\|x^{*}\right\|$ and the linearity of $x^{*}$, q. e.d.
The principal result of this section is

THEOREM 7. Let $\Phi$ be a continuous Young's function and $\Psi$ be its complementary function. Then

(a) To each $h \in B\left(S^{\Phi}(\mathscr{X}), \mathscr{Y}\right)$ there corresponds a unique $H$ in $W^{\Psi}(B(\mathscr{X}, \mathscr{Y}))$ such that

$$
h\left(F^{\prime}\right)=\lim _{\pi} \sum_{\pi} \frac{H\left(E_{n}\right)\left[F^{\prime}\left(E_{n}\right)\right]}{\mu\left(E_{n}\right)}
$$

for all $F \in S^{\Phi}(\mathscr{X})$.

(b) The correspondence $h \rightarrow H$ maps $B\left(S^{\Phi}(\mathscr{X}), \mathscr{Y}\right)$ linearly onto $W^{\Psi}(B(\mathscr{X}, \mathscr{Y}))$, and if $S^{\Phi}(\mathscr{X})$ is normed with $\|\cdot\|_{\Phi}$, then

$$
\|h\|_{B\left(S^{\Phi}(\mathscr{C}), \mathscr{Q}\right)}=\|H\|_{W^{\Psi}(B(\mathscr{X}), \mathscr{Y})} .
$$

Consequently, $B\left(S^{\Phi}(\mathscr{X}), \mathscr{Y}\right)$ and $W^{\Psi}(B(\mathscr{X}, \mathscr{Y}))$ are isometrically isomorphic.

Proof. (a) Let the norm of $S^{\Phi}$ be the Orlicz norm \|\|$_{\Phi}$ and $h \in B\left(S^{\Phi}(\mathscr{X}), \mathscr{Y}\right)$. If $h=0$, then $H=0 \epsilon W^{\Psi}(B(\mathscr{X}, \mathscr{Y}))$ satisfies (a). So let $h \neq 0$. Lemma 1 establishes the existence of a unique $\mu$-continuous finitely additive $B(\mathscr{X}, \mathscr{Y})$-valued set function $H$ which satisfies $(B)$. To complete the proof of (a), it remains to show that $H \in W^{\Psi}(B(\mathscr{X}, \mathscr{Y}))$.

First assume that $\Psi$ is continuous (i.e., $\Psi(x)<\infty$ for $x<\infty$ ). Let $\varepsilon>0$ be given and $y^{*}$ in the unit ball of $\mathscr{Y}^{*}$ be fixed but arbitrary. For an arbitrary partition $\pi=\left\{E_{n}\right\}$ of $\Omega$, form the $\mathscr{X}$-valued step function $G=\sum_{\pi} x_{n} \mu \cdot E_{n}$ where the $x_{n} \epsilon \mathscr{X}$ are subject to:

$$
\begin{aligned}
& \text { (i) } x_{n}=z_{n} \psi\left(\frac{\left\|y^{*} H\left(E_{n}\right)\right\|}{\|h\| \mu\left(E_{n}\right)}\right), \psi=\Psi^{\prime}, \\
& \text { (ii) The } z_{n} \in \mathscr{C} \text { satisfy } y^{*} H\left(E_{n}\right)\left(z_{n}\right)=\left\|y^{*} H\left(E_{n}\right)\right\|, \\
& \text { (iii) }\left|\Phi\left(\left\|z_{n}\right\| \psi\left(\frac{\left\|y^{*} H\left(E_{n}\right)\right\|}{\|h\| \mu\left(E_{n}\right)}\right)\right)-\Phi\left(\psi\left(\frac{\left\|y^{*} H\left(E_{n}\right)\right\|}{\|\hbar\| \mu\left(E_{n}\right)}\right)\right)\right|<\frac{\varepsilon}{m \mu\left(E_{n}\right)},
\end{aligned}
$$

where $m$ is the number of sets in $\pi$. The existence of such $z_{n}$ 's is ensured by lemma 5 and the continuity of $\Phi$. According to lemma 4 ,

$$
\begin{aligned}
1 & +I_{\Phi}(G) \geqslant\|G\|_{\Phi} \geqslant \frac{y^{*} h(G)}{\|h\|}, \quad \text { since }\left\|y^{*}\right\| \leqslant 1, \\
& =\frac{1}{\|h\|} y^{*} h\left(\sum_{\pi} x_{n} \mu \cdot E_{n}\right)=\frac{1}{\|h\|} \sum_{\pi} y^{*} h\left(x_{n} \mu \cdot E_{n}\right) \\
& =\frac{1}{\|h\|} \sum_{\pi} y^{*} H\left(E_{n}\right)\left[x_{n}\right], \quad \text { by the definition of } H \text { in lemma } 1, \\
& =\frac{1}{\|h\|} \sum_{\pi} y^{*} H\left(E_{n}\right)\left[z_{n}\right] \psi\left(\frac{\left\|y^{*} H\left(E_{n}\right)\right\|}{\|h\| \mu\left(E_{n}\right)}\right)
\end{aligned}
$$


by the choice of the $x_{n}$ 's,

$$
=\sum_{\pi}\left(\frac{\left\|y^{*} H\left(\mathbb{E}_{n}\right)\right\|}{\|h\|} \psi\left(\frac{\left\|y^{*} H\left(\mathbb{E}_{n}\right)\right\|}{\|h\| \mu\left(\mathbb{E}_{n}\right)}\right)\right)
$$

by the choice of the $z_{n}$ 's,

$$
=\sum_{\pi} \frac{\left\|y^{*} H\left(E_{n}\right)\right\|}{\|h\| \mu\left(E_{n}\right)} \psi\left(\frac{\left\|y^{*} H\left(E_{n}\right)\right\|}{\|h\| \mu\left(E_{n}\right)}\right) \mu\left(E_{n}\right) .
$$

Hence by equality in Young's inequality, we have $I_{\mathscr{\phi}}(G)+1$

(C)

$$
\sum_{\pi} \Phi\left(\psi \frac{\left\|y^{*} H\left(E_{n}\right)\right\|}{\|h\| \mu\left(E_{n}\right)}\right) \mu\left(E_{n}\right)+\sum_{\pi} \Psi\left(\frac{\left\|y^{*} H\left(E_{n}\right)\right\|}{\|h\| \mu\left(E_{n}\right)}\right) \mu\left(E_{n}\right) .
$$

But $G$ is a step function on $\pi$. According to theorem I.1.9,

$$
I_{\Phi}(G)=\sum_{\pi} \Phi\left(\frac{\left\|G\left(E_{n}\right)\right\|}{\mu\left(E_{n}\right)}\right) \mu\left(E_{n}\right)=\sum_{\pi} \Phi\left(\left\|z_{n}\right\| \psi\left(\frac{\left\|y^{*} H\left(E_{n}\right)\right\|}{\|h\| \mu\left(E_{n}\right)}\right)\right) \mu\left(\mathbb{E}_{n}\right)
$$

by the definition of $G$. Substituting this into (C) yields

$$
\begin{gathered}
1+\sum_{\pi} \Phi\left(\left\|z_{n}\right\| \psi\left(\frac{\left\|y^{*} H\left(E_{n}\right)\right\|}{\|h\| \mu\left(E_{n}\right)}\right)\right) \mu\left(E_{n}\right) \\
\geqslant \sum_{\pi} \Phi\left(\psi\left(\frac{\left\|y^{*} H\left(E_{n}\right)\right\|}{\|h\| \mu\left(E_{v}\right)}\right)\right) \mu\left(E_{n}\right)+\sum_{\pi} \Psi\left(\frac{\left\|y^{*} H\left(E_{n}\right)\right\|}{\|h\| \mu\left(E_{n}\right)}\right) \mu\left(E_{n}\right) .
\end{gathered}
$$

Hence

$$
\begin{gathered}
1+\sum_{\pi}\left|\Phi\left(\left\|z_{n}\right\| \psi\left(\frac{\left\|y^{*} H\left(E_{n}\right)\right\|}{\|h\| \mu\left(E_{n}\right)}\right)\right)-\Phi\left(\psi\left(\frac{\left\|y^{*} H\left(E_{n}\right)\right\|}{\|h\| \mu\left(E_{n}\right)}\right)\right)\right| \mu\left(E_{n}\right) \\
\geqslant \sum_{\pi} \Psi\left(\frac{\left\|y^{*} H\left(E_{n}\right)\right\|}{\|h\| \mu\left(E_{n}\right)}\right) \mu\left(E_{n}\right) .
\end{gathered}
$$

From the choice of the $z_{n}$ 's in (iii), it follows that

$$
\sum_{n} \Psi\left(\frac{\left\|y^{*} H\left(E_{n}\right)\right\|}{\|h\| \mu\left(E_{n}\right)}\right) \mu\left(E_{n}\right) \leqslant 1+\varepsilon
$$

Since the partition $\pi$ is arbitrary,

$$
I_{\Psi}\left(\frac{y^{*} H}{\|h\|}\right)=\sup _{\pi} \sum_{\pi} \Psi\left(\frac{\left\|y^{*} H\left(E_{n}\right)\right\|}{\|h\| \mu\left(E_{n}\right)}\right) \mu\left(E_{n}\right) \leqslant 1+\varepsilon .
$$

Since $\varepsilon>0$ is arbitrary, it follows that $I_{\Psi}\left(y^{*} H /\|h\|\right) \leqslant 1$. Therefore $y^{*} H \in V^{\Psi}\left(\mathscr{X}^{*}\right)$ and

$$
N_{\Psi}\left(y^{*} H\right) \leqslant\|h\| .
$$

Since $y^{*}$ was arbitrary in the unit ball of $y^{*}$, it follows that $H \epsilon W^{\Psi}(B(\mathscr{X}, \mathscr{Y}))$ and

$$
\|H\|_{w^{\Psi}}=\sup _{\left\|y^{*}\right\| \leqslant 1} N_{\Psi}\left(y^{*} H\right) \leqslant\|h\| .
$$

This proves (a) in the case that $\Psi$ is continuous. If $\Psi$ jumps at $M>0$, the same proof will be applicable provided it is shown that

$$
\Psi \frac{\left\|y^{*} H(E)\right\|}{\|h\| \mu(E)} \leqslant M
$$

for any $E \epsilon \Sigma_{0}$ and $y^{*}$ in the unit ball of $y^{*}$. Let $E$ and $y^{*}$ be fixed but arbitrary subject to these conditions. If $\mu(E)=0$, then $H(E)=0$, and the quotient above becomes $0 / 0=0$ by convention. If $\mu(E)>0$ and $x \in \mathscr{X}$ is arbitrary, then

Now

$$
\left|y^{*} H(E)[x]\right|=\left|y^{*} h[x \mu \cdot E]\right| \leqslant\left\|y^{*} h\right\|\|x \mu \cdot E\|_{\Phi} .
$$

$$
\|x \mu \cdot E\|_{\Phi}=\sup _{N_{\psi(}(\dot{A}) \leqslant 1} \sup _{\pi} \sum_{\pi} \frac{\left\|x \mu\left(E \cap E_{n}\right)\right\|}{\mu\left(E_{n}\right)}\left\|G\left(E_{n}\right)\right\| .
$$

In order that $N_{\Psi}(G) \leqslant 1$, it must be true that $\|G(E)\| / \mu(E) \leqslant M$ for all $E \in \Sigma_{0}$ (otherwise $I_{\Psi}(G / K)=\infty$ for $K \leqslant 1$ ). Hence

$$
\begin{aligned}
\|x \mu \cdot E\|_{\Phi} & \leqslant \sup _{\pi} \sum_{\pi}\left\|x \mu\left(E \cap E_{n}\right)\right\| M \\
& =\sup _{\pi} \sum_{\pi}\|x\| \mu\left(E \cap E_{n}\right) M=M\|x\| \mu(E) .
\end{aligned}
$$

Therefore

$$
\begin{aligned}
\left|y^{*} H(E)[x]\right| & \leqslant\left\|y^{*} h\right\| M\|x\| \mu(E) \\
& \leqslant\left\|y^{*}\right\|\|h\| M\|x\| \mu(E) \leqslant\|h\| M\|x\| \mu(E),
\end{aligned}
$$

since $\left\|y^{*}\right\| \leqslant 1$. Whence

$$
\left\|y^{*} H(E)\right\| \leqslant \sup _{\|x\| \leqslant 1}\|h\| M\|x\| \mu(E)=\|h\| M \mu(E) .
$$

Accordingly, $\left\|y^{*} H(E)\right\| /\|h\| \mu(E) \leqslant M$, which completes the proof of (a).

(b) To show the correspondence $h \rightarrow H$ is onto $W^{\Psi}(B(\mathscr{X}, \mathscr{Y}))$, let $H \in W^{\Psi}(B(\mathscr{X}, \mathscr{Y}))$ be arbitrary. An $h \in B\left(S^{\Phi}(\mathscr{X}), \mathscr{Y}\right)$ will be defined such 
that it corresponds to $H$. Define $h$ on all step functions $F_{\pi} \in S^{\Phi}(\mathscr{X})$ by

$$
h\left(F_{\pi}\right)=\sum_{\pi} \frac{H\left(E_{n}\right)\left[H^{\prime}\left(E_{n}\right)\right]}{\mu\left(E_{n}\right)}
$$

It is easy to see that $h$ is linear on the step functions. Moreover, if $y^{*} \epsilon y^{*}$ and $\pi=\left\{E_{n}\right\}$ is a partition of $\Omega$, then

$$
\begin{aligned}
\left|y^{*} h\left(F_{n}\right)\right| & =\left|y^{*} \sum_{\pi} \frac{H\left(E_{n}\right)\left[T\left(E_{n}\right)\right]}{\mu\left(E_{n}\right)}\right|=\left|\sum_{\pi} \frac{y^{*} H\left(E_{n}\right)\left[W^{\prime}\left(E_{n}\right)\right]}{\mu\left(H_{n}\right)}\right| \\
& \leqslant \sum_{\pi} \frac{\left\|y^{*} H\left(E_{n}\right)\right\|\left\|W^{\prime}\left(E_{n}\right)\right\|}{\mu\left(E_{n}\right)} \leqslant N_{\psi}\left(y^{*} H\right)\left\|W^{\prime}\right\|_{\infty}
\end{aligned}
$$

by theorem I. 1.21. (a). Hence

$$
\left\|h\left(F_{\pi}\right)\right\|_{y}=\sup _{\left\|\nu^{*}\right\| \leqslant 1}\left|y^{*} h\left(F_{\pi}\right)\right| \leqslant \sup _{\left\|y^{*}\right\| \leqslant 1} N\left(y^{*} \cdot H\right)\left\|F^{\prime}\right\|_{\Phi}=\|H\|_{w^{2}}\|F\|_{\infty},
$$

and $h$ is bounded. Now,

$$
\begin{aligned}
\left\|h\left(F_{\pi_{1}}\right)-h\left(F_{\pi_{2}}\right)\right\| & =\left\|h\left(F_{\pi_{1}}-F_{\pi_{2}}\right)\right\| \leqslant\|H\|_{W}\left\|F_{\pi_{1}}-F_{\pi_{2}}\right\|_{\phi} \\
& \leqslant\|H\|_{w^{\phi}}\left(\left\|F_{\pi_{1}}-F^{\prime}\right\|_{\phi}+\left\|H^{\prime}-W_{\pi_{2}}\right\|_{\phi}\right) .
\end{aligned}
$$

Therefore by IV. 3 and I. 19 (b)

$$
\lim _{\pi_{1}, \pi_{2}}\left\|h\left(F_{\pi_{1}}\right)-h\left(F_{\pi_{2}}\right)\right\|=0 .
$$

Thus $\lim h\left(F_{x}\right)$ exists strongly in $\mathscr{Y}$, and we define $h\left(F^{\prime}\right)$ for $F \in S^{\Phi}(\mathscr{X})$ by ${ }^{\pi}$

$$
h(F)=\lim _{\pi} \sum_{\pi} \frac{H\left(E_{n}\right)\left[F\left(E_{n}\right)\right]}{\mu\left(E_{n}\right)} \quad\left(=\lim _{\pi} h\left(F_{\pi}\right)\right) .
$$

Computations which are the same as those above show

(E)

$$
\left\|h\left(F^{\prime}\right)\right\| \leqslant\|H\|_{\mathrm{W}^{w} \|}\left\|F^{\prime}\right\|_{\phi} .
$$

Hence $h \in B\left(S^{\Phi}(\mathscr{X}), \mathscr{Y}\right)$. Olearly $H(E)=h(\cdot \mu \cdot E)$. Thorefore $h$ is mapped into $H$ by the correspondence of (a).

Now, the correspondence $h \rightarrow H$ from $B\left(S^{\mathscr{D}}(\mathscr{X}), \mathscr{Y}\right)$ onto $W^{\mathscr{X}}(B(\mathscr{X}, \mathscr{Y}))$ is obviously linear. Moreover, if $h \rightarrow H$, then. (D) yields $\|H\|_{\mathrm{W}^{\prime}} \leqslant\|h\|$ and (E) yields $\|h\| \leqslant\|H\|_{\mathrm{W}^{T r}}$. Thus $\|h\|=\| H_{\mathrm{W}{ }^{\prime} \|}$, q. e. d.

An immediate consequence of theorem 6 is that the conjugate space to $S^{\Phi}(\mathscr{X})$ is $V^{\Psi}\left(\mathscr{X}^{*}\right)$ since $W^{\Psi}(B(\mathscr{X}, \mathscr{Y}))$ collapses to $V^{\mathscr{X}}(B(\mathscr{X}, \mathscr{Y}))$ in the case where $\mathscr{Y}=\mathscr{C}$, the scalars.

CoRollary 7. Let $\Phi$ obey the $\Delta_{2}$ condition and ox be reflexive. Then theorem 6 remains true if $S^{\Phi}(\mathscr{X})$ is replaced by $V^{\Phi}(\mathscr{X})$.

Proof. Under these hypotheses $S^{\Phi}(\mathscr{X})=V^{\Phi}(\mathscr{Y})$, q. o.d.
COROLLARY 8. If $\Phi$ and its complementary functions $\Psi$ each obey the $\Delta_{2}$-condition, and $\mathscr{X}$ is reflexive, $V^{\mathscr{\Phi}}(\mathscr{X})$ is reflexive.

Proof. Two applications of corollary 7 yield the result, q. e. $d$

Applying these results to the $L^{\Phi}(\mathscr{X})$ spaces, one has the following corollary:

Corollary 9. Let $\Phi$ be a continuous Young's function and $\Psi$ be its complementary function, and $\mu$ have $F S P$, then

(a) to each $h \in B\left(M^{\Phi}(\mathscr{X}), \mathscr{Y}\right)$, where $M T^{\Phi}(\mathscr{X})$ is the closed subspace of $L^{\Phi}(\mathscr{X})$ determined by $\mu$-simple functions, there corresponds a unique $H \in W^{\Psi}(B(\mathscr{X}, \mathscr{Y}))$ such that

$$
h(f)=\lim _{\pi} \sum_{\pi} \frac{H\left(E_{n}\right)\left[\int_{E_{n}} f d \mu\right]}{\mu\left(E_{n}\right)}
$$

for all $f \in M^{\Phi}(\mathscr{X})$.

(b) The correspondence $h \rightarrow H$ maps $B\left(M^{\Phi}(\mathscr{X}), \mathscr{Y}\right)$ linearly onto $W^{\Psi}(B(\mathscr{X}, \mathscr{Y}))$ and if $M^{\Phi}$ is normed with $\|\cdot\|_{\Phi}$, then

$$
\|h\|_{B\left(M^{\Phi}(\mathscr{X}), \mathscr{S}\right)}=\|H\|_{W^{\Psi}(B(\mathscr{X}, \mathscr{Q}))} .
$$

Consequently $B\left(M^{\Phi}(\mathscr{X}), \mathscr{Y}\right)$ and $W^{\Psi}(B(\mathscr{X}, \mathscr{Y}))$ are isometrically isomorphic.

(c) If $\Phi$ obeys the $\Delta_{2}$-condition, $M^{\Phi}(\mathscr{X})$ may be replaced by $L^{\Phi}(\mathscr{X})$ in (a) and (b).

Proof. From the definitions of $M^{\Phi}(\mathscr{X})$ and $S^{\Phi}(\mathscr{X}), \lambda$, the isometric isomorphism of theorem II. 5 , maps $M^{\Phi}(\mathscr{X})$ onto a dense subset of $S^{\Phi}(\mathscr{X})$ isometrically by theorem I. 6 . The result immediately follows from theorem 6, corollary 7, and theorem III.9.

Remark 1. A specialization of this result shows that $\left(M^{\Phi}(\mathscr{X})\right)^{*}$ is isometrically isomorphic to $V^{\Psi}\left(\mathscr{X}^{*}\right)$ and when $\Phi$ obeys the $\Delta_{2}$-condition $\left(I^{\Phi}(\mathscr{X})\right)^{*}$ is equivalent to $V^{\Psi}\left(\mathscr{X}^{*}\right)$.

Remark 2. Employing the general bilinear vector integral of Bartle [3], we see that the representation

$$
h(f)=\lim _{\pi} \sum_{\pi} \frac{H\left(E_{n}\right)\left[\int_{E_{n}} f d \mu\right]}{\mu\left(E_{n}\right)}
$$

talkes the form $h(f)=\int_{\Omega} f d H$.

The referee has kindly brought the author's attention to the interesting papers of Albrycht and Orlicz $[1,2]$ which contain certain results related to the earlier part of this work. Unfortunately, the author was not aware of these papers at the time of the preparation of this work. However the work of $[1,2]$ and the present paper largely complement each other. 


\section{References}

[1] A. Albrycht and W. Orlicz, A note on modular spaces TI, Bull. Acad. Pol. Sci. 10 (1962), p. 99-106.

[2] - A note on modular spaces III, ibidem 10 (1962), p. 153-157.

[3] R. G. Bartle, A general bilinear vector integral, Studia Matl. 15 (1956), p. $337-352$.

[4] W. M. Bogdanowioz, Integral representation of linear continuous operators from the space of Lebesgue-Bochner summable functions into any Banach space, Proc. Nat. Acad. Sci. U. S. A. 54 (1965), p. 351-353.

[5] S. Bochner, Additive set functions on groups, Ann. of Math. 4:0 (1039), p. $769-799$.

[6] S. Bochner and R. S. Phillips, Additive set functions and vector laltioes, ibidem 42 (1941), p. 316-324.

[7] S. Bochner and A. E. Taylor, Linear functionals on cortain spaces of abstractly valued functions, ibidem 39 (1938), p. 913-944.

[8] L. E. Dubins and L. J. Savage, How to gamble if you must, Now York 1965. 1958.

[9] N. Dunford and J. T. Schwartz, Linear operators, Part I, Now York

[10] J. L. Kelley, General topology, Princeton 1955.

[11] M. A. Krasnosel'skii and Ya. B. Rutiokii, Oonvex funotions and Orlio spaces (Translation), Groningen 1961.

[12] K. Krickeberg and C. Pauc, Martingates et dérivation, Bull. Soc. Math. France 91 (1965), p. 455-544.

[13] S. Leader, The theory of $L^{p}$-spaces for finitely additive set functions, Aun. of Math. 58 (1953), p. 528:543.

[14] W. A. J. Luxemburg, Banach funotion spaces, Thesis, Delft 1955.

[15] M. Morse and W. Transue, Functionals $\Gamma$ bilinear over the product of two pseudo-normed vector spaces, II. Admissible spaces A, Ann. of Math. 51 (1950), p. $576-614$.

[16] R. A. Phillips, On wealtly compact subsets of a Banach space, Amer. J. Math. 65 (1943), p. 108-136.

[17] - On linear transformations, Trans. Amer. Math. Soc. 2 (1940), p. 516-541.

[18] M. M.. Rao, Linear functionals on Orlicz spaces, Nieuw Arch. Wisk. 12 (1964), p. $77-98$.

[19] - Linear functionals on Orlioz spaces (II), to appear.

[20] - Decomposition of vector measures, Proo. Nat. Aoad. Soi. U. S. A. 51 (1964), p. 771-774.

[21] J. J. Uhl, Jr., Orlioz spaces of finitely additive set funotions, linear opera. tors, and martingales, Bull. Am. Math. Soo. 73, in print.

[22] G. Weiss, A note on Orlioz spaces, Portugaliae Math. 15 (1956), p. 35-47.

[23] K. Yosida, Functional analysis, Berlin 1965.

[24] A. C. Zaanen, Linear analysis, Amsterdam 1953.

OARNEGIE INSTITUTE OF TECHNOLOGY

PITTSBURG, PENNSYLVANIA, U. S.A.

Regu par la Rédaction lo 28. 8. 1960

\section{The spectrum of an infinite product measure}

by

\section{R. KAUFMAN (Urbana, Ill.)}

The infinite product of certain probability measures on compact abelian groups was discussed by Varopoulus [4]; the measures he considered are included in the present description. We are mainly interested in showing how the orthogonality criterion of Kakutani [1] may be used in place of the almost-everywhere-convergence calculations of [4]. Besides this we give some elementary facts which aid in constructing examples in the harmonic analysis of measure algebras.

0. Let $G_{1}, G_{2}, G_{3}, \ldots$ be compact abelian groups $\neq 0, e_{n}$ the unit measure at 0 in $G_{n}, m_{n}$ the normalized Haar measure of $G_{n}$. Let $0<a_{n}<1$ for $1 \leqslant n$ and $\mu_{n}=a_{n} e_{n}+\left(1-a_{n}\right) m_{n}$. Finally,

$$
G=\prod_{1}^{\infty} G_{n}, \quad \mu=\prod_{1}^{\infty} \mu_{n} .
$$

THEOREM 1. The Fourier transform $\hat{\mu}$ vanishes at infinity in $\Gamma=\hat{G}$, if and only if $a_{n} \rightarrow 0$ (Varopoulos [4], p. 3806).

THEOREM 2. The measure $\mu$, as an element of the complex Banach algebra $M(G)$, has purely real spectrum if and only if

(i) $\sum_{a_{n}>1 / 2}\left(1-a_{n}\right)<\infty$.

(ii) For some integer $k \geqslant 1, \sum_{a_{n} \leqslant 1 / 2} a_{n}^{k}<\infty$.

1. The proofs are divided into one paragraph for the first theorem, and two for the second.

Proof of Theorem 1. It is well-known that a continuous character of $G$ is composed in an obvious way from a finite number of characters $\gamma_{1}, \gamma_{2}, \ldots, \gamma_{s}$ on $G_{1}, G_{2}, \ldots, G_{s}$ respectively, and that $\hat{\mu}$ takes the value $\prod_{1}^{s} \hat{\mu}\left(\gamma_{s}\right)$ on the composite character. This degenerates to $\Pi^{\prime} a_{n}, \Pi^{\prime}$ being the product over non-trivial components $\gamma_{n}$. It is clear that if

$$
a<\limsup _{n \rightarrow \infty}\left|a_{n}\right|<b \text {, }
$$

